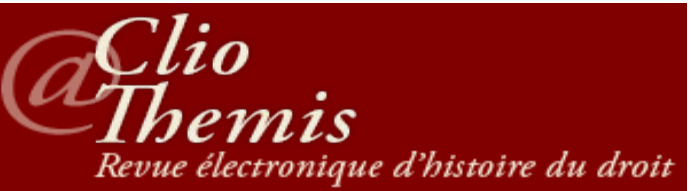

\title{
Le voyage retour de l'argent : la notion de rechange chez les juristes de la seconde modernité
}

\begin{abstract}
Résumé : Par l'intermédiaire de la notion de rechange qui contribue à l'organisation du voyage de l'argent, l'objectif de cet article est d'interroger l'analyse par les juristes des phénomènes économiques. Les mutations juridiques de la seconde modernité sont propices à un apport nouveau, mais les intérêts en jeu mettent à l'épreuve leur appréhension de la réalité des affaires. Entre la complexité des marchés et la régulation du voyage retour de l'argent, l'équilibre reste à trouver.
\end{abstract}

\begin{abstract}
Through the concept of recambium, which contributes to organize the travel of money, the main purpose of this article is to question the analysis by jurists of economic phenomena. The legal changes of the second modernity are conducive to a new contribution but the interests at stake test their understanding of business reality. Between the complexity of markets and the regulation of the return journey of money, a balance must be found.
\end{abstract}

Mots-clés : Lettre de change - Rechange - Juristes - Seconde modernité - Pensée économique.

Keywords : Bill of exchange - Recambium - Jurists - Second modernity - Economic thought.

Je vous prie de ne nous point remettre sur l'incertitude des pensions de M. de La Garde, et de nous envoyer une lettre de change. M. Colbert est un peu malade; si vous saviez ce qu'on fait de ce prétexte, même pour votre pension, vous verriez bien que rien n'est tel qu'une lettre de change ${ }^{1}$.

I. La lettre du 2i août 1676 de Madame de Sévigné à sa fille, Madame de Grignan, montre à quel point la lettre de change s'impose dès le XVII siècle dans les échanges quotidiens. Chez les négociants, elle est depuis la fin du Moyen Âge un outil incontournable de leur arsenal, dont l'épanouissement s'accélère pendant l'époque moderne.

2. En effet la lettre de change revêt une polyvalence qui satisfait les besoins renouvelés du commerce. Traditionnellement, le titre assure la mise à disposition de fonds d'une place sur une autre. Dans ce cadre, le donneur de valeur fournit une somme d'argent à une personne et reçoit, en échange, un instrument exécutoire du contrat, un engagement payable dans un autre lieu et dans une autre monnaie. Cette différence des lieux et des monnaies est à l'origine du prix du change et de sa légitimité. Le bénéfice ou la perte résulte de la différence des cours des changes entre les deux places, ce qui encourage les stratégies pour identifier les circuits les plus lucratifs. Toutefois, la diversité des monnaies tend à disparaître pendant la seconde modernité. En son absence, seul le jeu de l'offre et de la demande justifie l'écart plus ou moins important entre l'argent mis à disposition et la valeur du titre, d'autant que le travail fourni pour effectuer des remises de traites ou de fonds permet une majoration du prix. Mais la lettre de change est aussi un succédané de monnaie fiduciaire particulièrement prisé. Elle dynamise le marché des changes puisqu'elle assure tant le règlement des transactions que l'octroi d'un délai de paiement ${ }^{2}$. Les besoins en papier-

I Lettres de Madame de Sévigné, t. 5, nouvelle éd., Paris, Hachette, I862, p. 30.

2 Pour une présentation synthétique des fonctions de la lettre de change à l'époque moderne, voir O. Descamps et R. Szramkiewicz, Histoire du droit des affaires, $2^{\mathrm{e}}$ éd., Paris, LGDJ, 20I3, p. 22I. 
monnaie sont ainsi en mesure de faire augmenter ou baisser le trafic de lettres d'une destination à une autre.

3. Ce système de circulation des capitaux repose donc sur le triptyque suivant : la mise à disposition d'un moyen de paiement, l'utilisation d'un instrument crédit et la spéculation sur les changes. Il dépend, en partie, des possibilités de profit entre les places commerçantes et de la stabilité de la valeur des traites dans le commerces.

4. Le rechange intervient dans ce cadre pour assurer le transport de l'argent après un défaut de paiement d'une lettre de change ${ }^{4}$. Il se manifeste par une nouvelle remise de fonds, négociée chez un banquier, et l'émission d'une seconde lettre qui doit remplacer la premières. Ce mécanisme est indispensable dans le cadre d'une économie où « l'argent évidemment anime en même temps des circuits de plus large ouverture et aboutit, d'ordinaire, à un point central d'où il repart pour recommencer $»^{6}$. Sans l'effet de règlement, et malgré la protection cambiaire, le risque de rupture dans la circulation de l'argent peut altérer la stabilité des cours des changes et la confiance accordée dans ce système. Ces deux impératifs sont renouvelés par la généralisation de l'endossement pendant la seconde modernité ${ }^{7}$; or le voyage retour pallie la mise à disposition avortée de fonds et conserve la solidité du circuit financier.

5. À partir du XVIe siècle et jusqu'à la fin du XVIII siècle, ce mécanisme doit composer de plus en plus avec des exigences - parfois contradictoires - de sécurité et de profit dans le cadre d'une économie globalisée ${ }^{8}$. Les grandes découvertes ouvrent de nouvelles voies commerciales pour l'Europe à travers des marchandises inédites, le développement des moyens de communication, l'exploration de nouveaux territoires et l'afflux de métaux précieux. L'expansion à l'échelle mondiale d'un commerce animé par une caste de marchands-banquiers et d'armateurs entreprenants se concrétise et entraîne une intensification des échanges, tout en bouleversant l'ensemble de la sociétés. La conjoncture déplace ainsi la puissance économique de la Méditerranée au Nord, au service du grand commerce transatlantique, après une hégémonie passagère de la péninsule ibérique ${ }^{\text {Io }}$. Les trafics coloniaux et européens sont soutenus par un développement croissant de la banque et des techniques de crédit, dont la lettre de change est l'illustre représentante. Si la banque d'Amsterdam devient un pôle d'attraction des capitaux au XVIIe siècle aux côtés de Hambourg" l'Angleterre qui inaugure, au XVIII ${ }^{e}$ siècle, sa révolution industrielle et financière ${ }^{12}$. Ces mutations

3 Pour une vision synoptique de ces deux piliers du système de circulation des capitaux, voir J.-Y. Grenier, L'économie d'Ancien Régime. Un monde de l'échange et de l'incertitude, Paris, Albin Michel, 1996, p. 28. Pour une vision circonstanciée de ce phénomène, voir J.-G. Da Silva, Banque et crédit en Italie au XVII siècle, t. I, Paris, Éditions Klincksieck, 1969, p. 1617.

4 H. Lévy-Bruhl, Histoire de la lettre de change en France aux XVII -XVIII siècles, Paris, Recueil Sirey, 1933, p. 284.

5 M.-T. Boyer-Xambeu, G. Deleplace et L. Gillard, Monnaie privée et pouvoir des princes. L'économie des relations monétaires à la Renaissance, Paris, Éditions du CNRS, 1986, p. 39.

6 F. Braudel, Civilisation matérielle, économie et capitalisme, XVe-XVIII siècle, t. 2, Paris, Armand Colin, 1979, p. 73.

7 L'endossement permet, par signature au dos du titre, de donner l'ordre au tiré de payer à un nouveau bénéficiaire. Cette technique offre un règlement accéléré des créances et permet un nouveau change, donc une nouvelle spéculation. Sur la question de l'endossement et de sa propagation, voir R. De Roover, L'évolution de la lettre de change (XIV'-XVIII' siècle), Paris, Armand Colin, 1953, p. 83-II8 et H. Lévy-Bruhl, «L'endossement des lettres de change aux XVII et XVIII siècles », Annales de droit commercial français, étranger et international, 4, 1930, p. 253-284.

8 M.-T. Boyer-Xambeu, G. Deleplace et L. Gillard, « Du métal à l'espèce et du change à la banque », Cabiers d'économie politique. Monnaie métallique et monnaie bancaire, 18, 1990, p. 130.

9 F. Delleaux, Histoire économique de l'Europe moderne : XV'-XVIII' siècle, Paris, Armand Colin, 20I5, p. 34 et suivantes.

Io Ibidem, p. 9r et suivantes.

II Sur ces questions, voir notamment M. Weber, L'éthique protestante et l'esprit du capitalisme, Paris, Plon, 1985 ; C. Marsilio, «The Genoese exchange fair and the Bank of Amsterdam : Comparing two Financial Institutions of the $17^{\text {th }}$ Century », História Econômica ES História de Empresas, I8/I, 2015, p. $39-59$ et J.-G. Van Dillen, « La banque d'Amsterdam », Revue d'histoire moderne, 3/15, 1928, p. 16I-187.

I2 G. Oostindie et J. Vance Roitman, Dutch Atlantic Connections, I680-I8oo. Linking empires, bridging borders, Leiden et Boston, Brill, 20I4, p. 3 et suivantes. L'Angleterre est au XVIII siècle la terre d'élection de l'escompte, technique de mobilisation de créances utilisant le système du change tiré. 
s'accompagnent toutefois de nombreux désordres politiques et économiques : l'Europe est le théâtre de conflits constants et de perturbations monétaires récurrentes jusqu’au XVIII ${ }^{e}$ siècle $^{13}$.

6. Ainsi, l'époque moderne voit se former une mosaïque de places bancaires aux techniques affutées, assurant un flux de traites et de remises qui alimente l'Europe en papiers. Dans ce domaine, la France accuse un retard sensible à cause de troubles intérieurs ${ }^{14}$, elle demeure largement dépendante des réseaux bancaires extérieurs. Toutefois, des tentatives d'actualisation des structures commerciales et financières émergent et se cristallisent sous l'État centralisateur et absolutiste de Louis XIVis. À travers la multiplication des représentants de la puissance publique et le contrôle de la question marchande, la France s'insère peu à peu dans un esprit mercantiliste qui favorise la rénovation de ses circuits, de ses marchés de capitaux, et inaugure une courte période de stabilité monétaire ${ }^{16}$. Sous le règne de Louis XV, l'économie politique prend sa place dans la gouvernance ${ }^{17}$. Les préoccupations liées au négoce international et aux échanges de longue distance ${ }^{18}$ se traduisent par d'audacieuses tentatives pour assurer l'hégémonie commerciale du pays ${ }^{19}$, notamment en ce qui concerne la souplesse des mécanismes économiques.

7. Ce panorama renouvelle sans cesse les problématiques liées à la circulation de l'argent, d'autant que ces mutations atteignent rapidement le domaine juridique. En effet, la réglementation du négoce subit une profonde transformation, si ce n'est une renaissance. L'ordonnance de $1673^{20}$, élaborée à l'initiative de Colbert (1619-1683), inaugure l'entreprise de réforme du droit commercial et remodèle le paysage juridique français ${ }^{21}$. Cette nouvelle législation embrasse le voyage de l'argent par lettre, ainsi que son voyage retour, en deux temps : dans un titre 5 consacré à la lettre de change et aux billets de commerce, dans un titre 6 consacré aux intérêts du change et du rechange ${ }^{22}$. Cet ensemble de règles, qui entend avant tout intégrer le commerce dans l'ordre juridique étatique, n’est pas sans conséquences sur le monde des juristes.

I3 Concernant les conflits politiques qui secouent le XVII siècle, de la guerre de Trente Ans à la guerre de Hollande, voir par exemple F. Delleaux, op. cit., p. 78. Pour les guerres qui se multiplient au XVIII siècle, voir G. Daudin, Commerce et prospérité : la France au XVIII' siècle, Paris, Presses de l'Université Paris-Sorbonne, 2005, p. 2I4. À propos des désordres économiques, voir T. Luckett et P. Lachaier (trad.), « Crises financières dans la France du XVIII siècle », Revue d'bistoire moderne et contemporaine, 43/2, avril-juin 1996, p. 266-292.

I4 La France du XVII siècle subit une crise démographique et économique dont elle peine à se défaire, en plus du poids des conflits liés à la Fronde, L. Bély, La France au XVII siècle. Puissances de l'État, contrôle de la société, Paris, Presses Universitaires de France, 2009, p. 299 et suivantes, 337, 340-349 et 737.

I5 J. Imbert et H. Legohérel, Histoire de la vie économique ancienne, médiévale et moderne, Paris, Éditions Cujas, 2004, p. 423-423, 5 I3 et suivantes.

I6 O. Descamps et R. Szramkiewicz, op. cit., p. Irz et suivantes.

I7 A. Skornicki, «L'État, l'expert et le négociant: le réseau de la «science du commerce » sous Louis XV », Genèse, 65, 2006/4, p. 4-26.

I8 P. Butel, «Le négoce international en France au XVIII ${ }^{e}$ siècle », Le négoce international, XIII $-X X^{e}$ siècle, dir. F. Crouzet, Paris, Économica, 1989, p. 139-152.

I9 Par exemple, la mise en place du système de Law qui se solde par un échec en I719, les tentatives de libéralisation du secteur des grains et de la viande ou encore la réussite de la Caisse d'escompte à la fin du siècle, J. Imbert et H. Legohérel, op. cit., p. 507-508.

20 Pour une présentation générale de l'ordonnance, voir O. Descamps et R. Szramkiewicz, op. cit., p. I79-I84. Pour une analyse spécifique du texte sous l'angle de ses lacunes, voir J. Monéger, « De l'ordonnance de Colbert de I673 sur le commerce au code de commerce français de septembre 2000 : Réflexion sur l'aptitude du droit économique et commercial à la codification », Revue internationale de droit économique, I8, 2004/2, p. 17I-196; G. Bourcart, « Esquisse historique du droit commercial jusqu'au Code de commerce français de $1807 \gg$, Annales de droit commercial français, étranger et international, 33, I924, p. 259-283 et F. Terré, « Coutume et commerce », dans Le code de commerce, 1807-2007: livre du bicentenaire, dir. B. Teyssié, Paris, Dalloz, 2007, p. 46. À propos de l'influence de cette législation à l'étranger, on peut se référer à P.-L. Huvelin, L'bistoire du droit commercial : conception générale, état actuel des études, Paris, L. Cerf, 1904, p. 6o62, ou plus récemment C. Petit, «Derecho mercantil : entre corporaciones y còdigos », Hispania entre derechos propios y derechos nacionales, dir. B. Clavero Salvador, P. Grossi et F. Tomàs y Valiente, t. I, Espagne, Giuffrè Editore, 1990, p. 379380 .

2I Concernant le passage des ordonnances de réformation aux ordonnances de codification, voir J.-L. Harouel, «Ordonnances », Dictionnaire de la culture juridique, dir. D. Alland et S. Rials, Paris, Presses Universitaires de France, 2003, p. IIII-III3.

22 Voir le texte dans Isambert et al., Recueil général des anciennes lois françaises, t. 19, Paris, Belin-Leprieur, 1829, p. 92-I07. 
8. Ce dernier est marqué par une production doctrinale centrée sur les iura propria : provincial ou français, commentaire ou traité, recueil de jurisprudence ou de questions pratiques ${ }^{23}$. Sous l'impulsion de l'édit de Saint-Germain-en-Laye de 1679 , réformant l'enseignement du droit, on assiste à l'essor d'un droit français soutenu par des auteurs profondément marqués par le foisonnement législatif de cette époque, des ordonnances de Colbert à celles de d'Aguesseau ${ }^{24}$. Alors que la question cambiaire, comme le droit commercial dans son ensemble, est longtemps restée l'apanage des milieux d'affaires et de quelques spécialistes de la matière, sa captation par la législation royale l'intègre pleinement dans la modernité juridique. Il est encore difficile de parler d'un engouement de la doctrine pour la matière commerciale. Mais elle bénéficie indiscutablement de l'enthousiasme pour les sources du droit français : coutumes et législations royales. Nombreux sont les juristes, pétris de méthodes en chantier depuis la fin du $\mathrm{XVII}^{e}$ siècle ${ }^{25}$, qui se penchent pour la première fois sur cette discipline par l'intermédiaire de commentaires de l'ordonnance de 1673 , de traités spécialisés et de dictionnaires de commerce ${ }^{26}$. À l'image de cette littérature juridique, et malgré l'attraction du droit français, les juristes recouvrent des réalités variées et fluctuantes entre le XVII et le XVIII ${ }^{e}$ siècle ${ }^{27}$. Pour notre étude, ils renvoient surtout à ceux qui concourent à l'activité doctrinale, à la mise à disposition d'un savoir juridique ${ }^{28}$. Cette acception permet d'englober une diversité professionnelle et culturelle, qui constitue cependant une catégorie sociologique plus ou moins unifiée ${ }^{29}$ pouvant éclairer les enjeux, sans cesse renouvelés pendant la fin de l’Ancien Régime, du rechange.

Io. La variété qui caractérise la production doctrinale française impose une vision d'ensemble des ouvrages et des auteurs qui s'intéressent au domaine mercantile, sans prétendre à l'exhaustivité d'autant qu'il est encore malvenu de parler de doctrine commercialiste ${ }^{30}$. Le corpus assemblé offre un panorama fidèle de

23 A. Wijffels, Introduction bistorique au droit, $2^{\mathrm{e}}$ éd., Paris, Presses Universitaires de France, 20I4, p. 233.

24 Sur ces questions, on peut se référer à J.-L. Thireau, Introduction historique au droit, $2^{\mathrm{e} e ́ d ., ~ P a r i s, ~ F l a m m a r i o n, ~ 2003, ~ p . ~} 25 \mathrm{I}$ et C. Chêne, L'enseignement du droit français en pays de droit écrit (I679-I793), Genève, Droz, 1982, p. I8o. Concernant la fonction de la doctrine et le changement de paradigme entre l'époque médiévale et moderne, voir J.-L. Thireau, « La doctrine civiliste avant le Code civil », La doctrine juridique, dir. A. Bernard et Y. Poirmeur, Paris, Presses Universitaires de France, 1993, p. I6 et 2I-26. À propos du phénomène de codification et de la place de la législation royale dans le droit privé, voir Idem, «Les arrêtés de Guillaume de Lamoignon : une œuvre de codification du droit français ?», Droits, Revue française de théorie juridique, 39, 2004/I, p. 53-68.

25 G. Cazals, «Doctrine et pensée juridique, XII -XVIII siècle. La tentation globalisante », L'Histoire du droit en France. Nouvelles tendances, nouveaux territoires, dir. B. d'Alteroche et J. Krynen, Paris, Classiques Garnier, 20I4, p. II2-II3. La seconde modernité est le théâtre de multiples transformations de l’activité doctrinale française, de la dissipation des écoles géographiques à la constitution d'un corpus de droit français permettant un traitement plus national des sources. Concernant les mutations méthodologiques de la fin du XVII et du début du XVIII siècle, voir Q. Epron, La manière française du droit. Contribution à l'bistoire des méthodes juridiques de l'Humanisme aux pré-Lumières, Thèse Droit Paris II, 2006, p. 314 et suivantes.

26 Sur l'appropriation du droit commercial par certains juristes de l'époque moderne, voir A. Mages, « La place du droit commercial chez nos juristes anciens (XVIe-XVIII siècle) », Mémoires de la Société pour l'Histoire du Droit, vol. 70, 20I3, p. 225-241. Concernant la naissance d'un embryon de doctrine commercialiste, voir J. Guyader, « Existait-il une doctrine commercialiste dans l'ancienne France? », La doctrine, op. cit., p. 80-84; J. Hilaire, Introduction bistorique au droit commercial, Paris, Presses Universitaires de France, 1986, p. 64-66 et S. Molinier-Potencier, « La naissance de la doctrine commercialiste en Italie et en France », Méditerranées. Revue du Centre d'études internationales sur la Romanité, 30/3I, 200I, p. II9-138. Enfin, pour envisager la consolidation de la doctrine commercialiste, V. Simon, « Le récit des origines du droit commercial : la doctrine commercialiste et l'argument historique », Revue trimestrielle de droit commercial, 2, 20I8, p. 293-305.

27 Les termes « juristes » ou « doctrine » seront pris alternativement pour désigner les auteurs choisis au titre de ceux qui écrivent sur le droit pour en expliquer le fonctionnement. C’est ici une facilité de langage qui rend plus aisée la démonstration, mais qui ne doit pas masquer la complexité de ces deux notions recouvrant des réalités différentes. Il faut donc se garder d'unifier et de lisser la production juridique et ses acteurs. Pour plus de précisions, on peut se référer à G. Goubeaux, «Il était une fois... la Doctrine », Revue trimestrielle de droit civil, avril-juin 2004, p. 239-250; A. Sériaux, «La notion de doctrine juridique », Droits, Revue française de théorie juridique, 20, 1994, p. 65-74.

28 Ibid., p. 65 et 67.

29 «Leur formation universitaire, leur culture tant juridique que politique, leur expérience professionnelle leur confère une compétence sans pareille en la matière et un regard nécessaire éclairant, ce qui donne autorité et légitimité à leurs propos », M.-L. Duclos-Grécourt, L’idée de loi au XVIII siècle dans la pensée des juristes français (I7I5-I789), Paris, Presses universitaires juridiques de l'Université de Poitiers, Poitiers, 20I4, p. 22. Également sur ce sujet, G. Cazals, «Doctrine et pensée juridique $\gg$, art. cit., p. 95 -II5.

30 A. Mages, art. cit., p. 227. Depuis l'ordonnance de 1673 la discipline commence à se former chez nos juristes anciens, mais ces derniers n'effectuent que très rarement un traitement différencié du droit commercial. 
cette variété formelle et substantielle, tout en recouvrant l'ensemble de la période considérée ${ }^{31}$. Il permet d'envisager la façon qu'ont les juristes d'appréhender la notion de rechange pendant la seconde partie de l'époque moderne, tout en prenant en compte leurs parcours différents, leurs méthodes parfois distinctes sans être opposées.

II. Ces précisions nous incitent alors à présenter sommairement les ouvrages retenus à travers trois catégories principales qui constituent l'essentiel de la production doctrinale du moment ${ }^{32}:$ les commentaires des ordonnances de Jacques Savary (I622-1690) ${ }^{33}$, Philippe Bornier (1634-I7II) 34 , François de Boutaric (I672-1733) $)^{35}$, Daniel Jousse (1704-I78I) ${ }^{36}$ et Jacques-Antoine Sallé (I7I2-I778) ${ }^{37}$, embrassant les évolutions liées à l'enseignement du droit français et au processus de codification; les monographies d'Étienne Cleirac (1583-1657)38, Jacques Toubeau (1628-1685)39, Jacques Dupuis (deuxième partie du XVII siècle-première partie du XVIII siècle) ${ }^{40}$, Robert-Joseph Pothier (I699-I772) $)^{41}$ et Rogue ${ }^{42}$, qui choisissent de se consacrer à l'ensemble de la discipline commerciale ou à la question du change; le dictionnaire de commerce de Louis-Philémon Savary (1654-1727) et de Jacques Savary des Bruslons (1657I716) ${ }^{43}$, qui regroupe les principales notions en usage dans le commerce.

I2. À la frontière entre l'École, le Palais et le comptoir, professeurs de droit français ou praticiens témoins des difficultés dans l'application du droit, nul doute que cet ensemble reflète le caractère composite des réflexions que les juristes peuvent produire dans le domaine commercial ${ }^{44}$.

3I Cette diversité est nécessaire pour mesurer la quantité et la qualité de la production doctrinale. De plus, elle permet de cerner les nuances qui peuvent exister entre les auteurs sur un même sujet. Elle a pu être mise en œuvre par exemple chez M.-L. Duclos-Grécourt, op.cit., p. 28. Voir également G. Cazals, «Une renaissance. Doctrines, littérature et pensée juridique du XVI siècle en France », Clio@Themis, I4, 20I8, http://www.cliothemis.com/Une-Renaissance-Doctrine. L'auteur monte parfois en généralité à propos de la doctrine d’Ancien Régime, évoquant la difficulté de cerner l'ensemble d'une génération d'auteurs et l'utilité d'en retenir une tendance représentative ou originale.

32 Concernant la prééminence des commentaires et des traités spécialisés, voir J.-L. Thireau, op. cit., p. 259-263. À propos de l'engouement pour les dictionnaires, voir J.-C. Perrot, « Les dictionnaires de commerce au XVIII siècle », Revue d'bistoire moderne et contemporaine, 28/I, janvier-mars 198I, p. 36-67. Pour une vision plus circonscrite au droit commercial, on renvoi à J. Hilaire, Le droit, les affaires et l'histoire, Paris, Économica, 1995, p. 28-30.

33 J. Savary, Le parfait négociant, Paris, L. Billaine, i675. Voir également la réédition commentée d’É. Richard (éd.), Le parfait négociant, t. I, Genève, Droz, 2011.

34 P. Bornier, Conférences des ordonnances de Louis XIV, t. 2, nouvelle éd., Paris, les Associez, I755.

35 F. de Boutaric, Explication de l'ordonnance ou édit du mois de mars 1673, Toulouse, Gaspard Henault et Jean-François Forest, 1743 .

36 D. Jousse, Nouveau commentaire sur l'ordonnance du commerce du mois de Mars 1673, nouvelle éd., Paris, Debure l'âné, I76I.

37 J.-A. Sallé, L'esprit des ordonnances de Louis XIV, t. 2, Paris, Samson, 1758.

38 É. Cleirac, Usance du négoce ou commerce de la banque des lettres de change, Bordeaux, Guillaume de La Court, 1656 , l'ouvrage de cet avocat bordelais constitue un référent pré-ordonnance de 1673.

39 J. Toubeau, Les institutes du droit consulaire, t. 2, $2^{\mathrm{e}}$ éd., Paris, Nicolas Gosselin, 1700.

40 J. Dupuis, L'art des lettres de change, Paris, Arnoul Seneuze, 1693.

4I R.-J. Pothier, Traité du contrat de change, t. 2, Paris, et Orléans, Debure père et veuve Rouzeau-Montaut, 1768.

42 Rogue, Jurisprudence consulaire et instruction des négociants, t. 2, Angers, J. Jahyer, 1776.

43 J. Savary Des Bruslons et P.-L. Savary, Dictionnaire universel de commerce, t. 3, nouvelle éd., Paris, veuve Estienne, I74I.

44 Certains se sont imposés et ont traversé la Révolution de 1789 comme Boutaric, Bornier, Dupuis, Jousse, Pothier et Savary, M. Lesné-Ferret, «Des “commercialistes” du XIX siècle à l'écoute de Jousse », Daniel Jousse. Un juriste au temps des Lumières (I704-I78I), dir. C. Leveleux-Teixeira, Limoges, Presses Universitaires de Limoges, 2007, p. I7I-I72. D’autres sont plus discrets mais constituent tout de même des références en leurs temps : Rogue, Sallé ou Toubeau sont cités de nombreuses fois par leurs contemporains. Enfin, des auteurs sont oubliés malgré leurs qualités comme Cleirac, F. Trivellato, «La naissance d'une légende : juifs et finance dans l'imaginaire bordelais du XVII ${ }^{\mathrm{e}}$ siècle », Archives Juives, 47/2, 2014, p. 47-76. 
13. Malgré de vastes lacunes historiographiques ${ }^{45}$, le rechange relève ici deux intérêts majeurs. Le premier est évidemment la gestion des circuits du voyage de l'argent et des stratégies d'enrichissement qui y sont liées. Le second est celui du point de vue des juristes français offrant une grille analytique pertinente pour identifier la latitude laissée aux acteurs du négoce dans l'organisation de ce voyage retour. Néanmoins, leurs observations doivent être estimées par rapport aux caractéristiques du rechange et aux ambitions politiques et économiques de l'ordonnance de 1673 , dont la place ne doit pas être réduite. En effet, cette législation véhicule le discours mercantiliste de la monarchie absolue ${ }^{46}$, tout en étant le produit des évènements qui rythment cette période ${ }^{47}$. De ce fait, le rechange est une notion cruciale pour le développement économique de la France puisqu'il contribue à favoriser ou à restreindre, notamment à l'international, la circulation de l'argent et le financement des activités commerciales. Il est indispensable de déterminer sa place dans les écrits des juristes chargés d'en expliquer le fonctionnement.

I4. Surtout, en présence d'une législation à l'idéologie bien précise, le travail effectué par une partie de la production doctrinale de la seconde modernité permet de saisir la pertinence du régime juridique du rechange ou, au contraire, son dépassement. Cette étude offre également une plus grande visibilité sur l'écriture des juristes en matière commerciale : les auteurs que nous avons choisis ont-ils les outils pour appréhender le voyage retour de l'argent, alors que ce dernier répond avant tout aux échanges internationaux et aux marchés des changes?

I5. Il appert que le rechange, une fois placé sous l'égide d'un droit national, stimule l'expertise de nombreux juristes. Ils mettent à profit leurs méthodes, tant sur la forme que sur le fond, pour comprendre la logique à l'origine de cette technique, mais surtout de sa réglementation (I). Toutefois, les enjeux liés au voyage retour de l'argent dépassent amplement le cadre restrictif au sein duquel la notion est analysée. Malgré quelques tentatives pour souligner les problèmes issus de l'ordonnance de 1673 , les solutions offertes au négoce sont souvent lacunaires et peu originales. Les causes de cette faiblesse trouvent un écho dans les caractéristiques et les problématiques économiques de la circulation de l'argent qui échappent en partie à nos auteurs et, plus largement, au domaine juridique (II).

\section{Une approche pédagogique du rechange sous l'influence du droit français}

16. La propagation de la lettre de change dans le négoce, pour satisfaire les besoins en capitaux et le règlement intensif des transactions, rend particulièrement sensible la gestion du voyage de l'argent dans un contexte d'instabilité économique et politique. Les juristes de la seconde modernité relèvent avec acuité la complexité de cette question qu'ils présentent avec pragmatisme pour enseigner les différentes limitations qui affectent le rechange (B). Cette vertu didactique est avant tout le résultat d'une analyse rationnelle, systématique, qui permet d'inscrire la notion de rechange dans un cadre plus vaste, en accord avec les nouvelles relations qu'entretiennent les juristes et le droit commercial avec la législation royale $(\mathrm{A})$.

45 Les principales études relatives à la lettre de change ne recouvrent que très superficiellement cette notion. Par exemple, le rechange n'est mentionné que deux fois au titre du change fictif et de l'absence de paiement chez R. De Roover, op. cit., p. 28 et 34 . Une définition est faiblement esquissée chez H. Lévy-Bruhl, op. cit., p. 257 et 284. La notion n'est pas abordée dans l'importante étude de C. Carrière, M. Courdurié, M. Gutsatz et R. Squarzoni, Banque et capitalisme commercial. La lettre de change au XVIII siècle, Marseille, Institut historique de Provence, 1976.

46 C. Spector, « Le concept de mercantilisme », Revue de métaphysique et de morale, 39, 2003/3, p. $289-309$.

47 G. Bourcart, art. cit., p. 276-277. 


\section{A. L'inscription du voyage retour dans l'œuvre législative royale}

I7. La richesse de l'analyse des juristes ne peut se comprendre qu'en considération de l'ensemble dans lequel le rechange est placé. Après un titre 5 qui dresse le régime juridique de la lettre de change et du billet de commerce, le titre 6 de l'ordonnance sur les intérêts du change et du rechange consacre trois articles spécifiquement au voyage retour :

Article 4 : Ne sera dû aucun rechange pour le retour des lettres, s'il n'est justifié par pièces valables, qu'il a été pris de l'argent dans le lieu auquel la lettre aura été tirée ; sinon le rechange ne sera que pour la restitution du change avec l'intérêt, les frais du protêt et du voyage, s'il en a été fait, après l'affirmation en justice.

Article 5: La lettre de change, même payable au porteur, ou à ordre, étant protestée, le rechange ne sera dû par celui qui l'aura tirée, que pour le lieu où la remise aura été faite, et non pour les autres lieux où elle aura été négociée; sauf à se pourvoir par le porteur contre les endosseurs, pour le paiement du rechange des lieux où elle aura été négociée, suivant leur ordre.

Article 6: Le rechange sera dû par le tireur des lettres négociées pour les lieux où le pouvoir de négocier est donné par les lettres, et pour tous les autres, si le pouvoir de négocier est indéfini, et pour tous les lieux.

I8. Bien que la position des juristes face à la loi évolue du XVI ${ }^{e}$ au XVIII siècle, l'époque moderne est le témoin d'un courant nomophile qui se répand dans le paysage juridique français et qui contribue à gommer quelque peu les différences entre les littératures juridiques, mais aussi les auteurs ${ }^{48}$. Cette perspective se confirme chez les juristes, au titre du rechange, à travers trois voies principales : les préfaces, les épîtres dédicatoires et le plan. D’une part, la préface véhicule le motif de la publication, c'est un élément de compréhension de la démarche de l'auteur, alors que l'épître dédicatoire permet d'envisager l'appartenance à un réseau idéologique ${ }^{49}$. D'autre part, le plan reflète la conception retenue du rechange, l'approche choisie pour en expliquer le fonctionnement. Ainsi, ces deux éléments reflètent l'objectif et la logique suivis par l'auteur. L'exploration du péritexte ${ }^{\circ \circ}$ facilite l'appréhension du rechange dans son cadre pédagogique et rationnel d'étude de la matière commerciale, par des auteurs dont la formation est majoritairement juridique et résolument inscrite dans les mutations qui jalonnent les XVIIe et XVIIIe siècles.

19. De ce fait, on peut observer que certains reprennent in extenso le préambule de l'ordonnance de 1673 dans leurs propres préfaces, alors que d'autres se contentent de le reformuler. Cette attitude n'est pas sans conséquences puisque le préambule législatif dispose d'une valeur normative et idéologique importante, et témoigne de sa nature d'acte de gouvernement ${ }^{51}$.

20. Les commentateurs montrent la voie à emprunter « en suivant la logique initiée par les ordonnances de Colbert $\gg^{5^{2}}$. Le précurseur de ce mouvement n'est autre que Jacques Savary qui inaugure la démarche du commentaire en matière commerciale. Ce dernier ne camoufle pas son affection pour le texte de l'ordonnance en reproduisant ses grands principes. L'ancien mercier célèbre le bien-être du commerce, la

48 M.-L. Duclos-Grécourt, op. cit., p. 34-42. Ce constat fait écho aux progrès de la législation royale dans l'ordonnancement juridique et la littérature afférente, J.-L. Thireau, op.cit., p. 253-26I. Pour une mise en perspective historique, voir X. Prévost, «Mos Gallicus jura docendi. La réforme humaniste de la formation des juristes », Revue historique de droit français et étranger, 89/4, octobre-décembre 20II, p. 5II-512.

49 L. Kondratuk, «La réception d'un texte en histoire du droit: théorie littéraire et systématisation juridique », Revue bistorique de droit français et étranger, 98/I, janvier-mars 20II, p. I2.

so Ibid., p. II.

5I Sur la question de la valeur des préambules des ordonnances royales, voir D. Gaurier, « Les préambules des ordonnances françaises aux XVII et XVIII siècles : propagande royale ou véritable programme législatif ? », Légiférer, gouverner et juger. Mélanges d'histoire du droit et des institutions (IX ${ }^{e}-X X I^{e}$ siècle) offerts à Jean-Marie Cauchies à l'occasion de ses 65 ans, dir. E. Bousmar, P. Desmette et N. Simon, Bruxelles, Presses de l'Université Saint-Louis, 2016, p. 355-372 et F. SeignaletMauhourat, «La valeur juridique des préambules des ordonnances royales », Revue historique de droit français et étranger, 84/2, avril-juin 2006, p. 229-258. Voir également C. Dounot, « Le bien commun dans la législation royale (XIII ${ }^{e}$ XVIII ${ }^{e}$ siècle) », Bulletin de Littérature Ecclésiastique, 472/4, 2017, p. 99-II4.

52 A. Mages, art. cit., p. 234. 
lutte contre les abus et les fraudes ainsi que le maintien de la bonne foi dans le négoces3. Le rayonnement de la législation, qui est en grande partie due à la plume de Savary, offre à son ouvrage une dimension politique notamment lorsqu'il conclut, dans son épître dédicatoire, par un panégyrique de l'action colbertiste $^{54}$. Cette méthode trouve rapidement un écho favorable chez ses « disciples » qui n'hésitent pas à reproduire intégralement le préambule, sans ajout. C’est notamment le cas de Bornier, de Boutaric et de Salléss. L'enjeu de ces différents ouvrages est ainsi fixé, il faut se conformer à l'ordonnance de 1673, car elle entend assurer la prospérité du commerce et réguler l'activité mercantile.

2I. Les préfaces des traités sont plus variées. Dupuis exprime son intention de remédier aux lacunes de la doctrine et de la législation ${ }^{56}$. Il signale au passage la rigueur et la valeur du travail de Savary, dont il est un proche collaborateur ${ }^{57}$. Pothier, qui a pour habitude de s'intéresser à une diversité de sujets juridiques, se contente d'un article préliminaire pour présenter la notion de change et le plan de son étude ${ }^{8}$. Si son œuvre est en réalité un second tome intégré au traité de la constitution de rente, ce dernier ne déroge pas à la méthode du jurisconsulte. Enfin, Rogue consacre son épître aux juges-consuls du ressort d'Angers où il fut avocat et propose, en enseignant les lois du commerce, de réduire les litiges portés devant eux 5 .

22. Au regard de ces introductions aux commentaires de l'ordonnance ou à l'étude du change et des causes consulaires, une remarque doit être émise. En effet, chaque auteur, à sa façon, véhicule sa volonté d'offrir au lecteur un ouvrage didactique. Certains, comme Pothier, se concentrent sur l'appréhension de la matière ; d’autres, comme Dupuis ou Boutaric, interviennent en complément de l'action législative.

23. Cette dynamique est prolongée dans les plans qui structurent leurs analyses, car une grande majorité reprend la dichotomie de l'ordonnance et sa vision fragmentée des affaires ${ }^{60}$. En effet, la réglementation du voyage de l'argent sépare les trente-trois articles consacrés au régime juridique de la lettre de change et du billet de commerce des neuf articles qui régissent le prix du change et du rechange. Ce cloisonnement reflète la volonté de maîtriser le coût du rechange et se retrouve au sein des commentaires et des monographies avec plus ou moins de fidélité.

24. Les commentateurs sont évidemment ceux qui s'illustrent le mieux dans cette situation. Les plans adoptés reprennent la dualité entre le titre 5 et le titre 6 de l'ordonnance. C'est ce que l'on peut constater par exemple chez Savary et, quelques décennies plus tard, chez Sallé. Boutaric, Bornier et Jousse poussent la logique jusqu'à insérer chaque article de l'ordonnance avant de poser leurs observations ${ }^{6 \mathrm{I}}$. De cette façon, ils « s'adonnent tous à un commentaire, le plus souvent littéral, de chacun des articles et n'ajoutent rien aux matières abordées par le Code Savary dont ils respectent l'organisation générale ${ }^{62}$. Si dans le domaine du rechange l'idée d'exégèse pure semble un peu excessive, elle rend toutefois compte de la méthode à l'œuvre pendant la seconde modernité.

25. À titre de comparaison, Cleirac inscrit la question du rechange dans un chapitre consacré aux «Inconvéniens au refus d'acceptation \& à faute de payement ${ }^{63}$. Ce chapitre combine donc les développements liés à la protestation et ceux du voyage retour de l'argent ce qui reflète la chronologie des évènements. Cette logique se retrouve en apparence dans les traités de Dupuis et de Pothier, moins

53 J. Savary, op. cit., Préface.

54 Ibid., Épître.

55 P. Bornier, op. cit., p. 437-438 ; F. de Boutaric, op. cit., p. I-2 et J.-A. Sallé, op. cit., p. 335-337.

56 J. Dupuis, op. cit., Préface. La réédition de 1706 ne manifeste pas de changement substantiel. En revanche, celle de 1783 opte pour des développements plus larges et généraux sur les lettres de change.

57 F. Trivellato, The Promise and Peril of Credit, Princeton et Oxford, Princeton University Press, 2019, p. II5.

58 R.-J. Pothier, Traité du contrat, op. cit., p. I-3.

59 Rogue, op. cit., Épître. Concernant Toubeau, l'édition à laquelle nous avons accès contient une épître qui n'est pas le fait de l'auteur mais de son fils, J. Toubeau, op. cit., Préface.

60 J. Monéger, art. cit., p. 176 .

6I Nous renvoyons ici aux tables des matières des ouvrages cités.

62 A. Mages, art. cit., p. 237.

63 É. Cleirac, op. cit., p. 46-47. 
sensibles à la structure de la législation. Toutefois, cette originalité ne leur permet pas de tirer toutes les conclusions qui s'imposent sur le fond. Le rechange trouve ainsi sa place dans les obligations et droits qui découlent du voyage de l'argent. Cependant, il demeure limité aux prescriptions de l'ordonnance, c'est-àdire à la question du circuit et in fine de l'enrichissement. De la sorte, Dupuis inscrit le rechange dans les «droits du porteur d'une Lettre de Change protestée faute de payement ${ }^{64}$, mais son analyse ne se consacre qu'à la gestion des circuits et aux possibilités de profit. Pothier mentionne le rechange dans un chapitre consacré aux contrats qui interviennent dans la négociation des lettres de change, on reconnaît ici la rigueur du spécialiste des obligations. Le deuxième paragraphe qu'il accorde à ce domaine est dédié aux obligations du tireur, au titre desquelles nous retrouvons le rechange ${ }^{6 s}$. Pourtant, les développements sont intrinsèquement liés aux dispositions de l'ordonnance et à leur articulation : la question du prix du change, de son circuit, celle du rechange venant après.

26. L'organisation des traités des causes mercantiles de Toubeau et Rogue recoupe celle des commentaires et des monographies. Le premier inverse seulement la logique de l'ordonnance en dédiant son titre 5 au change et au rechange alors que son titre 6 concerne la lettre de change ${ }^{66}$. Le second place le change et le rechange entre le temps du paiement et la protestation, s'inscrivant dans la chronologie de l'opération.

27. Le rechange fait ainsi l'objet d'une appréhension à deux vitesses. Certains auteurs suivent la vie de la lettre de change, alors que d'autres isolent formellement la question de l'enrichissement. Cette différence ne doit pas masquer une démarche commune nonobstant la diversité des profils ${ }^{67}$. La logique derrière cette situation est difficile à identifier, mais elle témoigne indéniablement de la place prise par la législation royale et de la volonté des juristes d'en enseigner les prescriptions. En effet, « les praticiens plus ou moins expérimentés attendent de la doctrine une explication des textes législatifs qui modifieraient ou décideraient de leurs habitudes $\gg^{68}$. L'étude des préfaces, des épîtres dédicatoires et des plans, montre que les juristes de la seconde modernité cherchent à s'insérer dans les mutations du paysage normatif pour procurer une méthode rationnelle, afin de formuler les règles sociales et parvenir à un équilibre entre l'efficacité pratique des professionnels et la théorisation du droit ${ }^{69}$. Nous nous trouvons ici face à un aspect pédagogique important. Nos auteurs ont avant tout pour objectif de préciser la mise en ouvre de la réglementation du rechange, la meilleure façon de le faire n'est-elle pas d'adopter la logique de la législation correspondante? D’ailleurs, Boutaric avoue lui-même ne vouloir que se conformer aux prescriptions de l'ordonnance de commerce, rien de plus, rien de moins ${ }^{70}$.

28. Cette explication prend tout son sens pour les commentaires qui sont pleinement consacrés à l'étude des ordonnances et dont la méthode s'est forgée peu à peu à l'approche des textes. L'idée est de se détacher le plus possible des références extra-juridiques, peut-être à l'exception de Savary dont l'ouvrage est un véritable vade-mecum du négociant ${ }^{71}$. Une telle méthode n'est pas entièrement absente des monographies puisque le plan représente toujours, volontairement ou non, une certaine conception de la matière et du rôle fixé par l'écrit. En ce sens, les traités de la seconde modernité s'illustrent de plus en plus par une volonté de faire émerger les droits subjectifs des individus - ceux du tireur et du porteur dans notre cas avec des développements plus denses et précis, représentatifs de l'usus modernus français ${ }^{72}$.

\footnotetext{
64 J. Dupuis, op. cit., p. I89.

65 R.-J. Pothier, Traité du contrat, op. cit., p. 58 et suivantes.

66 Voir les tables des matières des deux traités.

67 L'exemple type est celui de Jacques Savary, car si ce dernier inaugure la méthode du commentaire de l'ordonnance du commerce, son profil est distinct des juristes comme Boutaric ou Bornier.

68 C. Chêne, op. cit., p. 196.

69 D. Baranger, Penser la loi, Paris, Gallimard, 2018, p. 267.

70 C. Chêne, op. cit., p. 167.

7I Toutefois, si cette assertion est vérifiée pour une grande partie de l'œuvre, le domaine du rechange est pauvre en digressions, voir infra $\$ 34$.

72 D. Baranger, op. cit., p. 290-300 et J.-L. Thireau, op. cit., p. 248-250 et A. Wijffels, op. cit., p. 233.
} 
29. Cette présentation reflète, à gros traits, la production doctrinale entre le XVI et le XVIII ${ }^{e}$ siècle et sapplique, non sans nuances ${ }^{73}$, aux auteurs choisis. Dès lors, ce rapprochement entre l'ordonnance et les ouvrages étudiés ne signifie pas forcément une adhésion à ses prescriptions et à son idéologie ${ }^{74}$. La place de la législation royale dans le commerce incite avant tout les juristes à adopter un raisonnement exégétique ${ }^{75}$, sans rejeter toute affiliation avec ladite source. C'est d'ailleurs l'exemple type de la production de Savary qui a pour objectif de promouvoir le texte et d'en combler les lacunes. Toutefois, il ne faut pas oublier le poids des stratégies éditoriales, du privilège de l'édition, du contrôle de la fabrication et de la diffusion des livres. Tous ces éléments incitent à se conformer aux attentes du pouvoir sous peine de censure ${ }^{6}$. De plus, certains auteurs n'octroient pas toujours à la législation un rôle central dans leur conception de l'ordre juridique, c'est notamment le cas de Pothier qui donne plus de force aux structures et engagements contractuels dans la formation et l'exécution des obligations ${ }^{77}$.

30. Il n'est donc pas aisé de déterminer si les juristes éprouvent un engouement pour l'ordonnance de 1673 ou s'ils s'y intéressent par pur conformisme. Néanmoins, il ne fait pas de doute que le rechange bénéficie des progrès méthodologiques de la doctrine de l'Ancien Régime. C'est ici un fait notable au regard de la

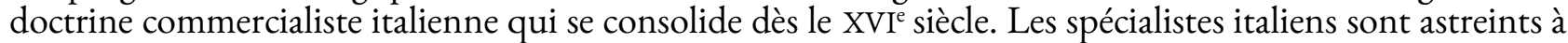
rassembler l'essentiel du droit des marchands pour en dessiner un exposé global des questions pratiques ${ }^{7}$, en raison d'un droit très composite qui se caractérise principalement par les usages et les réglementations locales. En France, le commerce est désormais soumis à une source nationale qui entend s’imposer dans le quotidien des praticiens. Partant, si le rechange est avant tout règlementé au regard de ses circuits et de son prix, ces deux dimensions doivent être le socle de l'activité doctrinale pour donner une explication pertinente de cette nouvelle réglementation, pour en éclairer les obscurités, pour en enseigner le respect, afin de soulager au mieux la vie du droit, quitte à en fragmenter la représentation ${ }^{79}$.

3I. L'apport des juristes pour l'analyse du rechange est incontestable sur la forme. La rationalisation de son étude, son appréhension dans un cadre structuré et presque unifié, s'inscrivant en parallèle de celui de sa source principale - l'ordonnance de 1673 - permet d'atteindre l'objectif que se fixe la majorité des auteurs : expliquer aux praticiens les enjeux et les impératifs de la norme qui s'impose à eux. Ce constat est intéressant car il rapproche des juristes aux profils très différents. Par exemple, Savary par son parcours professionnel et son expérience dans l'écriture de la loi est le mieux placé pour éclairer les commerçants sur les prescriptions de la législation ${ }^{80}$. Toubeau écrit son ouvrage pour encourager l'intégration, à titre autonome, du droit des marchands dans les sources juridiques nationales ${ }^{8 \mathrm{I}}$. Les professeurs de droit

73 Les nuances concernent notamment les auteurs en marge du système universitaire comme Savary, Toubeau ou encore Rogue.

74 La politique de Louis XIV consolide l'application de principes supérieurs au commerce, au service de la prospérité économique et dans un langage qui s'intègre au sein d'un esprit mercantiliste, ce qui justifie le contrôle des mouvements de capitaux, de marchandises et de personnes. Sur ces points, voir notamment A. Skornicki, « La France des Lumières et l'humanisme commercial. Bilan et perspectives historiographiques », Histoire, Économie É Société, 32, 2013/4, p. 79 ; L. Rothkrug, Opposition to Louis XIV : the political and social origins of the French Enlightenment, Princeton, Princeton University Press, 1965 , p. 38 et suivantes; C. Dounot, art. cit., p. I05.

75 J.-L. Thireau, « Droit national et histoire nationale : les recherches érudites des fondateurs du droit français », Droits, Revue française de théorie juridique, 38, 2003/2, p. $5 \mathrm{I}$.

76 S. Evrard, Le livre, le droit et le faux, Paris, L'Harmattan, 2017, p. 21-39.

77 Voir la préface de Jean-Louis Halpérin dans R.-J. Pothier, Traité des obligations, coll. Bibliothèque Dalloz, Paris, Dalloz, 2OII.

78 J. Hilaire, Introduction bistorique, op.cit., p. 65.

79 J.-L. Thireau, « Le jurisconsulte », Droits, Revue française de théorie juridique, 20, 1994, p. 29.

80 H. Hauser, «Le « Parfait Négociant» de Jacques Savary », Revue d'histoire économique et sociale, I3/I, I925, p. I-I8 et J. Hilaire, « Savary, Jacques », Dictionnaire historique, op. cit., p. 917-918.

8I J. Vendrand-Voyer, « Toubeau (Toubeau de Maisonneuve), Jean », Dictionnaire historique op cit., p. 973. 
français et avocats du XVIII ${ }^{\mathrm{e}}$ siècle comme Boutaric, Jousse, Sallé ou encore Pothier ${ }^{{ }_{2}}$, portent leur pierre à l'édifice du droit commercial dans le cadre de la réforme de l'enseignement du droit ${ }^{83}$.

32. Dès lors, nos auteurs s'inscrivent pleinement dans la réglementation du prix et des circuits du rechange. Si la forme préside le fond, la mise en ouvre du voyage retour impose d'en délimiter les contours et les circuits conformément à la législation de 1673 . À première vue, l'approche des juristes semble descriptive et linéaire. En réalité, elle relève avant tout d'une démonstration empirique qui caractérise la volonté des juristes modernes de se rapprocher de la pratique et d'offrir de véritables guides pour l'application des articles de l'ordonnance.

\section{B. La présentation pragmatique des restrictions du voyage retour}

33. À travers une série de dispositions aux articles 4 à 6 du titre 5, le Code Savary réglemente sévèrement le voyage retour. En effet, il doit s'organiser selon le premier circuit du change. Plus précisément, le prix du rechange ne peut être demandé au tireur que pour le lieu où la remise a été faite. Cette injonction marque un tournant majeur et trouve un écho favorable dans l'analyse de la notion par nos juristes de la seconde modernité.

34. Le bordelais Cleirac, dont l'ouvrage est publié avant l'ordonnance de 1673, permet de prendre conscience de cette évolution. Ce dernier parle simplement du fait de « prendre d'un autre Banquier autre Lettre de change pour semblable somme [...] à tel change qu'il trouvera $\gg^{84}$, sans mentionner les places qui constituent ce voyage retour. À l'inverse, les commentaires de l'ordonnance, tout comme les traités spécialisés, insistent sur la limitation du prix du rechange et s'emploient à en déterminer les frontières. Dès 1675, Savary inaugure cette nouveauté et définit le retour comme la traite prise du lieu où la première est protestée, sur celui d'où elle est tirée ${ }^{85}$. Quelques années plus tard, Dupuis s'intéresse également à ce changement de paradigme et souligne que, contrairement à l'ancien usage, le porteur doit désormais prendre une lettre de change dans la ville où la protestation a été faite ${ }^{86}$.

35. Contrairement à Cleirac, les auteurs post ordonnance inscrivent explicitement le circuit du rechange en symétrie de celui du change. Le retour doit s'effectuer depuis le lieu où la lettre n'a pas été payée, sur celui d’où elle a été tirée. Dans ce cadre, la question du prix devient centrale chez les juristes. Dupuis précise que le retour équivaut à la somme de la première lettre et comprend les frais du protêt, la provision, l'éventuel courtage ainsi que le prix payé pour assurer le retour de la traite ${ }^{87}$. Pour Pothier, le rechange s'effectue en tirant une lettre du lieu où la première a été tirée sur celui d'où elle a été émise et correspond à une même somme d'argent que le change initial ${ }^{88}$. Bornier ajoute que le retour doit s'établir uniquement entre la ville où l'on doit payer et celle où l'on demeure par une lettre de surprotêt, au change que l'on trouvera et pour la somme de la première lettre. Il ajoute d’ailleurs que ce nouveau change se fait toujours à perte pour le tireur $^{89}$. Cette notion de perte se retrouve également chez Boutaric qui distingue toutefois si le tireur est

82 Tous ces auteurs entretiennent certaines affinités. Par exemple, Jousse fait l'éloge de Pothier en tête de certains de ses ouvrages, voir la préface de J.-L. Sourioux dans Daniel Jousse., op. cit., p. II. Boutaric dispose d'une carrière similaire à celle du jurisconsulte orléanais qui, pour sa part, reconnaît en Dupuis un jurisconsulte aguerri, J. Poumarède, « Enquête sur un juriste au-dessus de tout soupçon: François de Boutaric (1672-1733), Droits, Revue française de théorie juridique, 40, 2004/2, p. 23-46 et M. Lesné Ferret, art. cit., p. 177. Il y a donc des inspirations potentielles entre ces différents auteurs du XVIII siècle.

83 Les ordonnances de codification, avec la création d'enseignements de droit français, contribuent au renouvellement de la pensée juridique moderne, C. Chêne, op. cit., p. 200.

84 É. Cleirac, op. cit., p. 56.

85 J. Savary, op. cit., p. II7.

86 J. Dupuis, op. cit., p. I90-I9I.

87 Ibid., p. I88-I89. Ce prix est appelé « nouveau change », ce qui permet de le lier au premier voyage de l'argent.

88 R.-J. Pothier, Traité du contrat, op. cit., p. 63.

89 P. Bornier, op. cit., p. 628. 
débiteur du porteur ou non ${ }^{\circ}$. Sallé va jusqu'à définir le rechange comme l'intérêt d'intérêt' , une conception originale que Toubeau conteste, ${ }^{22}$ mais qui met en valeur l'appréhension du voyage retour du point de vue de son coût.

36. Les auteurs abordent donc le rechange de deux façons: la première se concentre sur le circuit, la seconde sur le prix. Cette présentation peu originale a le mérite de refléter avec fidélité les prescriptions inédites de l'ordonnance. Les juristes nous enseignent que le rechange doit correspondre au circuit inverse $\mathrm{du}$ premier voyage et implique de payer un nouveau change auquel s'ajoutent différents frais. Tous insistent sur cette conception restrictive qui permet d'exclure les pratiques spéculatives et de conformer le prix du second change au premier.

37. Pendant la seconde modernité, la propagation du change tiré dans tout le négoce rend particulièrement sensible ce choix de limiter le voyage retour. Le jeu des changes n'est plus l'apanage de grandes firmes internationales, mais devient le quotidien des négociants qui emploient des traites pour leurs affaires. Il s'agit d'une portion non négligeable de l'activité économique dans son ensemble, permettant de potentialiser les profits de chaque transaction ${ }^{93}$. En conséquence, les manipulations susceptibles de faire fluctuer les cours des changes s'avèrent plus dangereuses puisqu'elles peuvent affecter généreusement le commerce.

D’ailleurs, dès la fin de l'époque médiévale, la ricorsa a largement encouragé la transformation des opérations de change en une pratique lucrative qui s’affranchit du circuit en droiture pour emprunter une place intermédiaire ${ }^{94}$. L'objectif est d'organiser le voyage retour en modifiant l'une des deux places d'origine pour trouver un meilleur taux et donc un change plus avantageux. Le calcul de la différence entre l'argent remis et l'argent reçu, c'est-à-dire le profit du cambiste lorsqu'il fournit de l'argent pour une lettre avec un change plus bas, ou lorsqu'il tire pour de l'argent reçu avec un change plus haut, devient un enjeu crucial dans ce cadre et favorise une circulation artificielle des lettres.

L'endossement est un acteur majeur de ce phénomène et justifie l'intervention du législateur dans le cadre de sa politique mercantiliste. La technique permet de faire passer une lettre de change de main en main et donc de multiplier les trajets intermédiaires avec un seul papier. Elle se répand en France, dès le début du $\mathrm{XVII}^{\mathrm{e}}$ siècle, et donne la possibilité de multiplier les manipulations sur l'argent en voyage, et donc les profits spéculatifs"s. Si l'utilité de l'endos pour le règlement en chaîne des créances est évidente, le risque de provoquer une inflation des cours des changes et de gêner le commerce dans ses besoins en moyens de paiement et de crédit l'est tout autant.

38. Malgré quelques lacunes, l'ordonnance de 1673 perçoit les abus qui peuvent résulter de la négociation des lettres de change par l'endos. Les articles 5 et 6 limitent ainsi le rechange au lieu où la négociation a été permise dans la relation entre le tireur et le porteur. Dès lors, le voyage retour est soumis au consentement des parties à l'opération, et non à la volonté d'un seul de multiplier ses gains ou de diminuer ses pertes. Nos auteurs sont sensibles à cette préoccupation et consacrent de nombreuses lignes à l'épineux problème de la négociabilité du titre. Face au caractère relativement récent de l'endossement, il s'agit d'une originalité qui doit être soulignée, même si son traitement reste à géométrie variable. En effet, la notion est totalement absente chez Cleirac qui ne fait référence qu'au circuit primitif du voyage retour. En revanche,

90 F. de Boutaric, op. cit., p. 76. Cette question de la relation entre le tireur et le porteur est déterminante, car elle va définir ce que chacune des parties doit supporter au titre des pertes.

9 I J.-A. Sallé, op. cit., p. 394.

92 J. Toubeau, op. cit., p. I83.

93 J. Bottin, « En quête de profits. La pratique des changes à Rouen et en Europe de l'Ouest (I580-1640) 》, Revue d'bistoire moderne et contemporaine, 63/I, 2016, p. 53-54 et L. Dermigny, « La banque à Montpellier au XVIII siècle », Annales du Midi : revue archéologique, historique et philologique de la France méridionale, 93/151, 1981, p. 2 I.

94 Sur la ricorsa, autrement appelée change avec recourse, voir $\mathrm{H}$. Lapeyre, « La banque, les changes et le crédit au XVI siècle », Revue d'Histoire Moderne ES Contemporaine, 3/4, octobre-décembre 1956, p. 295-296. Concernant les stratégies spéculatives sur le change, voir M.-T. Boyer-Xambeau, G. Deleplace et L. Gillard, op. cit., p. 38.

95 L'endossement n'est pas considéré comme une technique répandue en France avant le début du XVII siècle, H. Lévy-Bruhl, op. cit., p. IO3 et suivantes. 
l'hypothèse est omniprésente chez les autres. Il y aurait donc une prise de conscience à la suite de la codification Louis-quatorzième et de la diffusion des techniques cambiaires.

39. De cette façon, tous reconnaissent aux articles 5 et 6 de l'ordonnance la faculté de restreindre le rechange à la négociation autorisée ${ }^{96}$. Une explication avant tout contractuelle, d'inspiration civiliste, est avancée : la limitation s'impose pour respecter les principes qui lient les parties au contrat. Dupuis s'exprime en ces termes quand il énonce que l'obligation substantielle, celle qui résulte du contrat de change, est la promesse du tireur de payer le rechange en cas de protêt du lieu où elle a été tirée sur son lieu d'origine, et non pour chaque lieu où elle a été négociée par voie d'endossements successifs ${ }^{97}$. Cette position est assumée par Pothier qui insiste sur l'engagement du tireur à l'égard du bénéficiaire ${ }^{98}$. Jousse met lui aussi l'accent sur l'absence de participation du tireur, et donc le déséquilibre dans l'avantage perçu lors de la circulation du titre, pour conforter la limitation aux seules négociations autorisées 9 . Ainsi, l'objectif est, comme le rappelle clairement Boutaric, d'éviter de bouleverser la relation à l'origine du contrat de change ${ }^{\mathrm{roo}}$. Il faut empêcher que le bénéficiaire ne fasse courir la lettre de change de place à place pour augmenter ses chances de profit, cela au détriment du tireur qui doit rembourser le rechange. Les auteurs analysent donc cette limitation du point de vue des rapports entre les parties. Ils considèrent que le tireur ne peut être tenu au-delà de son engagement initial. Ce point de vue illustre l'ambivalence du change tiré qui oscille entre l'efficacité économique, notamment dans le cadre de la spéculation, et la sécurité nécessaire pour conserver l'utilité du titre dans les échanges quotidiens. En effet, la lettre de change s'affranchit de plus en plus de la relation originelle entre le tireur et le bénéficiaire en raison de sa négociabilité. Il en résulte une dépersonnification des rapports économiques qui impose de limiter la garantie solidaire à laquelle le tireur est tenu, au risque que toutes les opérations de change deviennent trop dangereuses pour lui.

40. Deux auteurs proposent une lecture alternative de la restriction, sans pour autant s'éloigner de la ligne directrice ainsi fixée. Dans son traité sous forme d'Institutes, Toubeau énonce la limitation du rechange, mais précise que le législateur «n’a pourtant pas voulu par la empecher la négociation des lettres de change »; au contraire, la loi entend seulement lutter contre les abus et les usures ${ }^{\text {10. }}$. Quelques années après la promulgation de l'ordonnance, cette conception se retrouve déjà chez Savary pour qui la restriction des articles 4 à 6 permet d'éviter que les banquiers ou les marchands agissent fictivement et nuisent au commerce ${ }^{102}$. On perçoit ici la sensibilité de ces spécialistes pour la cause commerciale.

4I. L'explication contractuelle, majoritaire chez nos auteurs, est aussi symptomatique de la place du contrat chez certains juristes de l'Ancien Régime. En effet, cette période est considérée comme l'âge du consensualisme, notamment grâce à l'école du droit naturel qui concrétise les avancées des canonistes de l'époque médiévale en la matière ${ }^{103}$. C'est ce qui explique cette distinction par exemple entre Pothier, fervent jusnaturaliste ${ }^{104}$, et Savary dont les préoccupations sont d’abord mercantiles. Plus généralement, on observe ici une distinction entre les auteurs du XVII siècle et ceux du XVIII siècle. D’ailleurs, Savary provient d'un milieu d'affaires et dédie toute sa carrière au négoce, alors que Toubeau ne troque jamais sa position à l'échevinage de Bourges. Ces derniers laissent l'explication consensualiste de côté, peut-être sontils trop contemporains pour pouvoir en profiter. Leur proposition, liée aux abus de la banque, parvient

96 Cette première idée est résumée en référence au travail de Bornier, Dupuis et Savary chez J. Savary Des Bruslons et P.L. Savary, op. cit., p. 1078.

97 J. Dupuis, op. cit., p. 199-200.

98 R.-J. Pothier, Traité du contrat, op. cit., p. 65-66.

99 D. Jousse, op. cit., p. 142.

Ioo F. de Boutaric, op. cit., p. 78. Cette affirmation prouve que l'ordonnance de 1673 ne permet pas aux juristes de dépasser cette distinction entre le contrat de change et la traite, le premier reste le socle de la relation entre le tireur et le bénéficiaire. Sur cette question voir M.-A.-M., Darwish, Évolution historique de la conception de la lettre de change. Étude comparée entre le droit français, le droit égyptien et le droit musulman, Thèse Droit Paris I, 1990, p. 191-194.

IoI J. Toubeau, op.cit., p. I85.

IO2 J. Savary, op. cit., p. 219-220.

103 J.-L. Gazzaniga, Introduction historique au droit des obligations, Paris, Presses Universitaires de France, 1992, p. I7I-176.

I04 J.-L. Thireau, « Pothier, Robert-Joseph », Dictionnaire bistorique, op. cit., p. 832-835 et J.-L. Sourioux, « Pothier ou le Sphinx d'Orléans », Droits, Revue française de théorie juridique, 39/1, 2004, p. 69-76. 
tout de même à procurer le même résultat et montre une volonté d'expliquer la légitimité des restrictions imposées par le législateur.

42. Nos auteurs semblent prendre fait et cause pour les enjeux de ces prescriptions et font preuve d'une vertu pédagogique qui doit nous intéresser. En effet, quel que soit le type de littérature juridique envisagé - commentaire ou monographie - les juristes présentent une science du droit empirique, pétrie d'une portée pratique sans être privée d'un esprit d'érudition ${ }^{105}$. D’ailleurs, la réforme de l'enseignement du droit encourage largement le concours des praticiens à l'ouvre doctrinale. Ce constat se maintient jusqu'au XVIII ${ }^{e}$ siècle $^{106}$ et permet l'apparition d'une génération de juristes qui « témoignent d'une connaissance approfondie des réalités du droit, d'un souci d’application concrète des règles juridiques ${ }^{107}$, tant au profit des étudiants que des professionnels.

43. Cet esprit pratique se manifeste sous diverses formes dont nous tenons ici un témoignage à travers les modèles et les illustrations du rechange. La majorité de nos auteurs s'inscrivent dans cette configuration qui contribue à rapprocher la production doctrinale issue de l'École, du Palais, mais aussi du comptoir pour la famille Savary ${ }^{108}$.

44. Les spécialistes de la question commerciale engagent le pas, comme Jacques Savary qui fournit une mise en situation au lecteur pour envisager une opération de change et de rechange dans un circuit Paris Bordeaux. Dans cet exemple, Pierre tire depuis Paris une lettre de 3000 livres sur Paul à Bordeaux. La lettre est payable à Jean et le prix du change s'élève à deux pour cent soit 60 livres. La lettre est protestée, Jean prend la même somme à un banquier de Bordeaux pour une traite dont le prix du change s'élève à 6o livres. Pierre, le tireur, doit restituer à Jean la somme de la lettre, les i2o livres du change et du rechange, en plus de différents frais ${ }^{109}$. Chez Rogue, l'illustration est plus légère, mais sans doute aussi plus claire. Il décrit la situation dans laquelle «si la lettre est tirée de Lyon sur Paris, on ne doit que le change de Paris à Lyon, \& non d'un autre endroit où il seroit plus considérable ${ }^{\text {ㅍ }}$. La méthode est aussi mise à contribution par les commentateurs. Par exemple, Boutaric nous soumet le cas de Pierre qui fournit à Paris une lettre de change de iooo livres sur Toulouse. À l'échéance, la lettre est protestée ce qui l'oblige de prendre une nouvelle lettre sur Paris, pour la même somme, avec le change et les frais qui en découlent ${ }^{\mathrm{II}}$.

45. Après avoir présenté des modèles de circuits du rechange, les auteurs n'oublient pas d'illustrer la limitation liée à l'endossement. Boutaric poursuit son exemple précédent, celui du banquier Pierre, qui ne doit le rechange que pour Toulouse sur Paris et non sur Bordeaux où la lettre a été négociée sans son accord, tous les autres rechanges étant le fait des endosseurs ${ }^{112}$. Toubeau est tout aussi éloquent. Son illustration concerne un marchand qui obtient une lettre de change de Paris sur Lyon, avec la faculté de la

Ios La distance entre l'étude et la pratique du droit a été largement nuancée dès l'humanisme juridique, voir X. Prévost, Jacques Cujas (I522-I590). Jurisconsulte bumaniste, Genève, Droz, 2015, notamment la seconde partie. À propos de cette nuance et du consensualisme, Id., «La réticence des humanistes envers le consensualisme », Revue des contrats, I, mars 20I5, p. I40. Pour la seconde modernité et la place des praticiens dans l'enseignement et la production doctrinale, voir J.L. Gazzaniga, «Quand les avocats formaient les juristes et la doctrine », Droits, Revue française de théorie juridique, 20, 1994, p. 3I-4I et G. Cazals, « Doctrine et pensée juridique », art. cit., p. II2-Ir3. Enfin, concernant la « culture pratique » chez les juristes de l'époque moderne, voir C. Chêne, «La place des professionnels dans la formation des juristes aux XVII et XVIII siècle », Annales d'bistoire des facultés de droit, 2, 1985, p. 5I-62.

Io6 D. Ribard, «L'écriture de la doctrine, XVII ${ }^{e}$ XVIII ${ }^{e}$ siècle », L'écriture des juristes, XVI'-XVIII siècle, dir. L. Giavarini, Paris, Éditions Classiques Garnier, 20ıо, p. II6.

I07 J.-L. Thireau, op. cit., p. 248.

Io8 Boutaric, Dupuis, Cleirac et Sallé ont été avocats. Jousse et Pothier ont occupé chacun une charge de conseiller au présidial d'Orléans. Bornier et Toubeau ont été respectivement lieutenant particulier près la cour du sénéchal de Montpellier et échevin. Nous renvoyons ici aux différentes notices correspondantes du Dictionnaire bistorique, op. cit. Pour Cleirac, voir L. Lamothe, Notes pour servir à la biographie des hommes utiles ou célébres de la ville de Bordeaux, Paris, I865, p. I4. Pour Dupuis, nous nous référons à la première approbation de l'édition de 1693.

I09 J. Savary, op. cit., p. 217.

IIo Rogue, op. cit., p. 397.

III F. de Boutaric, op. cit., p. 75. On peut aussi citer un modèle de rechange depuis la protestation chez É. Cleirac, op.cit., p. 5556. Également J. Dupuis, op. cit., p. I89.

II2 F. de Boutaric, op. cit., p. 77. 
négocier là où il aura besoin d'argent. Dans un tel cas, lors de la protestation, le tireur est soumis au rechange et à tous les changes successifs qui pourront avoir lieu par la négociation autorisée du titre, entre Paris et Lyon ${ }^{113}$. Dupuis se révèle adroit dans cet exercice avec l'exemple de Pierre de Paris qui remet à Jean une lettre de change de 3000 livres, tirée sur Paul de Lyon. La lettre est endossée par Jean au profit de Jacques d'Amsterdam, puis au profit de Bernardin de Venise, puis de Guillaume de Lyon. La lettre est protestée et, en l'absence d'autorisation générale de négocier, chaque endosseur doit rembourser le rechange à son endossataire, en remontant jusqu'au tireur et au bénéficiaire initial ${ }^{114}$.

46. Les différents circuits du rechange sont ainsi appréhendés, expliqués et illustrés. Une mise en action de la règle décrite par la norme législative permet d'inscrire le discours des juristes dans leur contexte. En effet, les modèles ne sont pas des hypothèses purement théoriques, mais correspondent à une réalité économique précise. Les villes mentionnées par les auteurs comme Paris, Lyon et Amsterdam représentent d'importantes places de change à l'échelle nationale et internationale ${ }^{\text {Is. }}$. De plus, elles sont accompagnées de différentes configurations pour rendre compte de la diversité des opérations, y compris au niveau du prix. Il est possible cependant de regretter un manque de détails sur la question monétaire et la conversion pour le change étranger, ou l'opportunité d'endosser une lettre sur certaines places.

47. Si le travail des juristes n'est pas aussi complet qu'on le voudrait, leurs explications sur la mise en ouvre d'une opération de rechange bénéficient d'une analyse soucieuse d'éclairer une législation peu loquace. Des auteurs comme Pothier, Jousse et Boutaric cristallisent cette volonté de mettre à disposition des guides pour respecter les exigences du droit français ${ }^{116}$. Savary, dont le profil est nettement différent, n'est pas à l'écart de cette génération de juristes, car son ouvre entière est dédiée à la profession mercantile dans un objectif pédagogique ${ }^{\text {Ir }}$.

48. Les progrès qui sont accomplis ne sont d'ailleurs pas isolés, puisque cette dynamique se retrouve dans d'autres disciplines commerciales comme la comptabilitér" ${ }^{\text {. }}$. Malgré cela, le travail des juristes n'est pas exempt de griefs. À gros traits, la méthode utilisée permet de faire parler le législateur et d'expliquer le fonctionnement du voyage retour de l'argent. Toutefois, la volonté des juristes d'effectuer une analyse systématique et pragmatique ne suffit pas à saisir les complexités du rechange dans le cadre d'une économie globalisée et en perpétuel renouvellement.

\section{Une prise en compte insuffisante des enjeux économiques du rechange}

49. Les intentions du législateur sur la circulation des capitaux sont claires et les juristes parviennent à en expliquer les principales conséquences. Cependant, ils peinent à appréhender les nombreuses lacunes qui concernent l'articulation des dispositions législatives avec les réalités des affaires $(\mathrm{A})$. Malgré des efforts pour produire des guides destinés aux praticiens, une grande partie des juristes ignore les rouages du commerce dont les enjeux ne sont pas toujours en adéquation avec ceux du droit (B). Sur ce point, le rechange est en retrait des progrès juridiques à l’œuvre durant la seconde partie de l'époque moderne.

\footnotetext{
II3 J. Toubeau, op.cit., p. 185-186.

II4 J. Dupuis, op. cit., p. 192-194.

IIs P. Jeannin, Marchands du Nord, espaces et trafics à l'époque moderne, Paris, Presses de l'École normale supérieure, 1996, p. 156 et 295 et J. Bottin, art. cit., p. 47-84.

II6 C’est évidemment ici l’apport majeur des professeurs de droit français, praticiens transformés en universitaires.

II7 É. Richard (éd.), Le parfait, op. cit., p. 23.

II8 X. Prévost, «Remarques historiques sur le formalisme comptable : les « livres et registres des négociants 》 (I673-I807) », Le formalisme. Sources et technique en droit privé positif. Actes du colloque organisé le 20 Octobre 2016 à l'université de Pau et des Pays de l'Adour, dir. N. Laurent-Bonne et S. Tisseyre, Paris, LGDJ, 2017, p. 166-170.
} 


\section{A. Une analyse lacunaire face aux besoins de la pratique cambiaire}

50. La réglementation du rechange montre rapidement ses limites face aux nécessités du commerce. Elle ignore parfois la vie du droit et des affaires par une volonté exacerbée de contrôler les flux de capitaux. À l'égard de ce constat, nos auteurs sont mauvais élèves, car si certains parviennent à dévoiler l'impasse à laquelle aboutit le formalisme imposé pour la mise en œuvre du voyage retour, l'ensemble demeure largement insuffisant.

5I. La symétrie entre le change et le rechange a pour objectif d'éliminer les abus liés aux procédés spéculatifs, tout en permettant un contrôle plus aigu de la circulation de l'argent. Cependant, la fixation d'un seul circuit retour peut se heurter à la complexité du réseau des changes et à la fluctuation de la conjoncture. Dans un tel cas, l'utilité de la réglementation n'est pas assurée au regard des besoins de la pratique cambiaire ${ }^{119}$ : les réseaux d'affaires, chaînes de crédit, établissements bancaires et le dynamisme économique sont autant de facteurs qui dirigent les circuits du change, du rechange, et qui exigent la flexibilité du voyage de l'argent.

52. Ainsi, il peut être difficile, voire inenvisageable, de tirer du lieu de paiement de la première lettre de change sur celui de son émission. C'est ici une critique, souvent discrète, qui est adressée par certains juristes à la législation. Dupuis est le premier à montrer que l'ordonnance est dépassée dès qu'il n'y a pas de négoce réglé et ordinaire entre les deux places qui sont imposées, lorsque le change ne se pratique pas ou que l'on ne trouve pas de fonds à tirer en respectant le circuit du change. Pour étayer son propos, il donne l'exemple de Boulogne et de Paris qui nécessitent un intermédiaire comme Lyon ${ }^{120}$. Plus simplement, Rogue précise que le rechange ne peut s'effectuer que s'il y a un « commerce ordinaire \& reglé entre les places d'où l'on tire \& celle où la lettre devoit être payée $\gg^{121}$, sans indication supplémentaire. Tout aussi laconique, Pothier se contente d'affirmer la légitimité d'un prix plus élevé pour le voyage retour, par le recours à une place de substitution lorsqu'il est impossible de respecter le circuit du change ${ }^{122}$.

53. Les auteurs sont donc peu nombreux à relever ce problème. Plus encore, Jousse admet qu'il est possible d'emprunter un lieu intermédiaire si l'on ne trouve pas de lettre sur le circuit du change initial, mais il milite pour un strict respect de l'ordonnance. Ainsi, l'équité exige que le porteur engendre le moins de perte pour le tireur. Dans un tel cas, il n'est pas permis de réclamer un rechange supérieur ${ }^{123}$. De cette façon, il exonère le tireur du supplément engendré par le retour en contradiction de l'article 4 de l'ordonnance, surplus qu'il considère comme illégitime.

54. La question du circuit de retour impossible fait donc l'objet d'un traitement lapidaire. Seuls trois auteurs se prononcent véritablement et l'un d'entre eux en dénie la légitimité. De plus, la différence entre la situation du tireur-débiteur et celle du tireur-commissionnaire n'est que peu relevée, elle est pourtant essentielle pour identifier lequel des deux doit rembourser le rechange, surtout en cas de négociation ${ }^{124}$. Ce constat se renforce avec la problématique du lieu de substitution pour organiser ledit circuit alternatif ; or cette hypothèse ne bénéficie pas de l'expertise des juristes en la matière.

55. En effet, Pothier se contente de fournir une ligne directrice dans l'appréciation du lieu de remplacement pour réaliser un rechange. Il considère que le porteur ne doit pas être réduit à l'impossible, mais qu'il doit

II9 Cette idée est développée par exemple chez M. Vigne, La banque à Lyon $d u X V^{e}$ au XVIII siècle, Lyon et Paris, Rey et Guillaumin, 1903, p. 99-I00; P. Pourchasse, Le commerce du Nord. Les échanges commerciaux entre la France et l'Europe septentrionale au XVIII siècle, Rennes, Presses universitaires de Rennes, 2006, p. 244 ou encore G. Daudin, op. cit., p. II8.

I20 J. Dupuis, op. cit., p. 194.

I2I Rogue, op. cit., p. 397.

122 R.-J. Pothier, Traité du contrat, op. cit., p. 64.

I23 D. Jousse, op. cit., p. I39-I40.

I24 En effet, le tireur-commissionnaire agit pour le compte du bénéficiaire. Ainsi, il doit s'exécuter au nom et avec les fonds de ce dernier. La question du remboursement du rechange ne fait donc pas débat, contrairement au tireur-débiteur qui ne peut être amené à rembourser plus que ce qu'il doit en raison de la créance détenue par le porteur. 


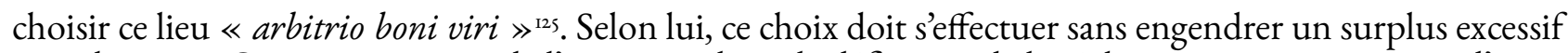
pour le tireur. Cette prescription de l'auteur souligne la déficience de la réglementation qui impose d’avoir recours à une solution transactionnelle dont le jurisconsulte orléanais a le secret ${ }^{126}$. Mais si la critique esquissée a le mérite d'offrir une réponse favorable aux besoins des affaires, elle demeure isolée. Malgré sa prise de position sur les insuffisances du texte, Dupuis ne désigne qu'une place tierce qui fait du négoce ordinaire et réglé avec les villes d'émission et de paiement du premier change ${ }^{127}$. Jousse reste fidèle à son opinion pour exclure tout surplus lors du rechange. Toutefois, il conseille le recours à des circuits nationaux qui offrent un change au pair ou favorable, comme le circuit Paris - Lyon dont les risques de perte pour le tireur sont très faibles, voire inexistants ${ }^{128}$.

56. L'absence de disposition en accord avec les réalités du voyage de l'argent compromet gravement les prescriptions de l'ordonnance. Il existe un véritable besoin en matière de flexibilité des opérations de change en raison du caractère international des échanges. La fluctuation des valeurs peut toucher un vaste ensemble de marchés; or l'existence d'importants réseaux entre places et maisons de commerce permet d'adapter les circuits et d'organiser avec plus de latitude le voyage de l'argent. Par exemple, les nombreuses crises internationales qui jalonnent le XVIII ${ }^{e}$ siècle ${ }^{129}$ sont atténuées par l'intermédiaire d'Amsterdam qui centralise une grande partie des opérations de change. Depuis la diaspora protestante, la ville regroupe de riches maisons de banque et des particuliers capables d'assurer le retour depuis le Nord pour minimiser les pertes ou maximiser les profits ${ }^{130}$. Ce constat se retrouve lors des pénuries d'espèces et de papiers. Elles sont régulièrement dénoncées par les négociants et affectent d'importantes villes de commerce comme Bordeaux et Marseille qui doivent trouver des places suffisamment alimentées en fonds pour organiser le

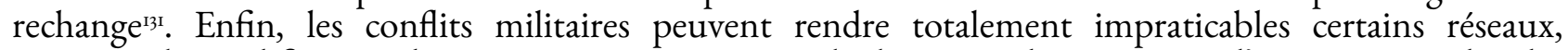
imposant la modification du voyage retour. Par exemple, la guerre de succession d'Espagne interdit, dès I702, l'émission ou la réception de traites entre la France et l'Angleterre, le passage par Amsterdam ou Genève est inévitable ${ }^{132}$. Ainsi, un rechange en vase clos peut nuire aux opérations en cours. La faculté d'organiser le retour sur d'autres places est salvatrice pour le bien-être du commerce. Les juristes expliquent peu cette possibilité qui semble condamnée par la législation royale : seulement trois d'entre eux y consacrent quelques lignes imparfaites. Leur analyse est donc insuffisante, car elle demeure trop tributaire de l'économie générale de l'ordonnance.

57. Cette situation se confirme dans les actes de la pratique, ce qui contribue à interroger l'efficacité de l'analyse du rechange par nos juristes, puisque la formule «à défaut, un protêt a été fait pour le change, rechange, dommages-intérêts, pour prendre la même somme sur une place où on en trouvera » est récurrente dans les protêts bordelais du XVIII siècle $^{133}$. Cette formule souligne la nécessité de recourir au circuit qui présente une offre de fonds suffisante, quel qu'il soit. La possibilité d'emprunter une voie intermédiaire est parfois attestée par les courtiers eux-mêmes, comme l'illustre la formule adressée à l'armateur bordelais Jean Pellet: «Je soussigné agens de change certifie a tous ceux a qui il apartiendra navoir peu trouver d'argeant dans cette place et sur icelle de Bordeaux que a un quard pour cent de perte aux lettres a vüe en foi de quoi j’ai signé le present $[\ldots] \gg^{134}$. Un tel document témoigne de l'importance des places de substitution, car le port aquitain n'offre que du change à perte.

\footnotetext{
I25 R.-J. Pothier, Traité du contrat, op. cit., p. 64-65.

I26 En effet, Pothier est animé par l'idée d'un droit juste qui laisse une large place aux solutions transactionnelles, modérées, assez souples et qui ménagent l'intérêt du commerce, J.-L. Sourioux, « Pothier ou le Sphinx d'Orléans », art. cit., p. 70-7I.

I27 J. Dupuis, op. cit., p. 194.

I28 D. Jousse, op. cit., p. I40.

I29 T. Luckett et P. Lachaier (trad.), art. cit., p. 266-292.

I30 G. Daudin, op. cit., p. I3I et P. Pourchasse, op. cit., p. 205 et 242.

I3I F.-X. Emmanuelli, La crise marseillaise de I774 et la chute des courtiers, Paris, Éditions du CNRS, I979, p. I et suivantes ; P. Butel, Les négociants bordelais, l'Europe et les «Isles » au XVIII siécle, Paris, Aubier Montaigne, 1974, p. 9 et suivantes.

132 H. Lüthy, La Banque Protestante en France, t. I, Paris, SEVPEN, 1959, p. I50.

I33 Cette mention est tirée d'un protêt du 20 mars 1709, dressé par le notaire bordelais Jacques Dufau, Archives Départementales de la Gironde, 3 E 507I.

I34 ADG, 7 B I8I7.
} 
58. Ainsi, l'adaptation est le maître mot du voyage retour de l’argent, mais les juristes sont peu réceptifs à ce besoin ce qui se confirme à propos de la question de la preuve. En effet, la protestation est une exigence traditionnelle pour requérir le remboursement du change et du rechange ${ }^{135}$, l'ordonnance ne fait que codifier ici un usage de longue date. Toutefois, dans son article 4 du titre 6 , elle impose une nouvelle condition probatoire qui ne facilite pas l'intelligibilité de la réglementation du voyage retour ${ }^{136}$. Toubeau insiste sur cette innovation et souligne que sous l'empire des anciennes ordonnances, quel que soit le circuit emprunté, seul le protêt permettait de condamner le tireur au change et au rechange ${ }^{137}$. Désormais, le législateur modifie cette configuration et sollicite la preuve, par des pièces valables, de la légitimité du voyage retour.

59. Cette exigence probatoire s'intègre parfaitement à la lutte contre les abus que Colbert s'est fixé. Néanmoins, la législation est lacunaire. Elle se veut concise sur la teneur de la preuve, tout en éludant son articulation avec la question de l'instabilité des circuits du change et du rechange. En conséquence, l'effectivité de la réglementation du voyage retour est remise en cause ${ }^{138}$ sans que les juristes ne parviennent à fournir d'explication réellement satisfaisante. En effet, la preuve est faiblement abordée dans leurs analyses qui se contentent de désigner un justificatif délivré par un banquier, un agent de change ou un négociant ${ }^{139}$. En dépit de cette discrétion, trois auteurs relèvent avec pertinence les conséquences de cette nouvelle injonction en distinguant désormais le droit de faire le rechange du droit de s'en faire rembourser. De ce fait, le protêt donne le droit au porteur de prendre de l'argent en retour, mais seules les pièces justificatives permettent de se retourner contre les signataires pour en obtenir le remboursement ${ }^{140}$.

6o. En outre, certains développements renferment de précieux conseils pour le négoce. Si l'on peut chercher en vain des modèles comme ceux fournis pour comprendre la circulation des traites, afin de se faire une idée plus précise des documents avec lesquels les négociants doivent se justifier, Dupuis et Boutaric semblent prendre en compte la question de la voie alternative en cas d'impossibilité de respecter ledit circuit. Le premier souligne bien que le certificat délivré peut justifier un circuit de substitution, il précise d'ailleurs qu'il s'agit du seul moyen de le légitimer ${ }^{141}$. Le second, sans affirmer explicitement cette légitimité, décide que le justificatif doit être présenté même si le porteur n’a pas « fourni des Lettres pour le lieu d'ou avoit été tirée la lettre protestée $\gg^{142}$. Ainsi, dans l'hypothèse d'un circuit différent, le respect de l'exigence probatoire permet toujours d'engager l'action en remboursement à l'encontre du tireur et des autres garants. Si ces deux solutions sont favorables au négoce, elles manquent cependant de cohérence face aux faibles explications sur les circuits alternatifs.

6I. Les juristes s'illustrent donc ici par d'importantes carences, ils n'offrent pas les solutions attendues face aux insuffisances de la législation. Malgré une tentative d'éclairer avec cohérence la nouvelle réglementation de 1673 , un décalage peut être souligné entre l'analyse du rechange et sa mise en œuvre par la pratique. Il serait toutefois excessif d'en conclure à une incompétence générale en matière commerciale tant les mutations juridiques de l'époque moderne sont à l'origine de très nombreux progrès accomplis par la doctrine. Par exemple, en matière de comptabilité, mais aussi de commandite ${ }^{143}$, de multiples innovations sont élaborées, comblant d'importantes lacunes de la législation. Une autre perspective se dévoile alors: le rechange semble être négligé par les réflexions juridiques et témoigne d'une difficile perméabilité entre le droit et le commerce.

I35 Voir notamment O. Descamps et R. Szramkiewicz, op. cit., p. 234.

I36 Pour une présentation intégrale de l'article, voir J. Savary Des Bruslons et P.-L. Savary, op. cit., p. I078.

I37 J. Toubeau, op. cit., p. I83.

I38 La notion d'effectivité s'entend ici du degré d'utilisation réelle de la norme, de la mesure du respect de son injonction, J.F. Perrin, Pour une théorie de la connaissance juridique, Genève, Droz, 1979, p. I28.

139 J. Savary, op. cit., p. 217 ; D. Jousse, op. cit., p. I4I et J.-A. Sallé, op. cit., p. 394.

I40 J. Savary, op. cit., p. 218 ; P. Bornier, op. cit., p. 268-269 et F. de Boutaric, op. cit., p. 76.

I4I J. Dupuis, op. cit., p. 203-207.

142 F. de Boutaric, op. cit., p. 75 .

143 Voir par exemple J. Hilaire, La société en commandite entre son passé et son avenir, dir. A. Viandier, Paris, Librairies techniques, 1983, p. I43 et suivantes et X. Prévost, « Remarques historiques sur le formalisme comptable », art. cit., p. I75I77. 


\section{B. Un discours juridique à l'écart des réflexions sur le commerce}

62. Le contexte de rédaction de l'ordonnance de 1673 est propice à une politique de contrôle du voyage de l'argent, notamment pour permettre le redressement des finances, des ressources économiques et militaires du pays ${ }^{144}$. Dans ce cadre, la pensée mercantiliste peut aisément exercer son joug sur le texte ${ }^{145}$ et soumettre le rechange à l'influence de Colbert : l'ingérence de l'État pour contrôler et orienter l'activité mercantilet46.

63. Néanmoins, de nombreuses pistes de réforme du commerce sont envisagées au XVIII siècle. Dans ce cadre, le rôle de l'argent est désormais débattu en faveur d'un assouplissement de la réglementation. Boisguilbert préfigure l'évolution des idées économiques à la charnière des XVII et XVIII siècle. Il est l'un des premiers penseurs à nuancer le mercantilisme sans totalement rompre avec lui ${ }^{147}$, considérant que les marchés doivent être dictés par une libre concurrence favorable à l'autorégulation ${ }^{148}$. Cette conception se retrouve quelques décennies plus tard chez Forbonnais. Ce précurseur de la physiocratie estime que la circulation de l'argent dépend du degré de liberté laissé aux acteurs économiques dans les processus de paiement, de crédit et de spéculation ${ }^{149}$.

64. Les néo-mercantilistes adhèrent à cette idée de libre circulation de l’argent pour améliorer la situation du commerce, ils placent dès lors l'État dans une position passive à cet égard ${ }^{\text {Iso }}$. L'avènement des physiocrates au cours du XVIII siècle pousse la logique à son extrême avec une conception agraire et libérale de l'économie. La notion de produit net prédomine et l'argent devient un simple moyen de dynamiser l'économie nationale, la liberté étant la clef de ce dynamisme ${ }^{151}$.

65. De cette façon, la mutation des idées économiques bouleverse les doctrines mercantilistes sur la circulation de l'argent ${ }^{152}$. Dès le milieu du XVIII siècle, le paradigme s'ancre dans le paysage européen, mais les juristes sont peu réceptifs à cette évolution. Consciemment ou non, la faible critique juridique s'accompagne d'une absence de remarque sur l'aspect économique de la réglementation du rechange.

66. En effet, les remises en cause des articles du titre 6 sont ténues, même lorsqu'il s'agit de souligner l'impossible respect des prescriptions de l'ordonnance. Dupuis fait figure de trublion dans la mesure où il

144 F. Lebrun, Le XVII siècle, 2e éd., Paris, Armand Colin, 2003, p. 248 et suivantes.

I45 Les guerres menées par Louis XIV favorisent cette « fringale monétaire, incoercible », D. Dessert, Argent, pouvoir et société au Grand Siècle, Paris, Fayard, 1984, p. 27 et D. Villey, Petite histoire des grandes doctrines économiques, $3^{\mathrm{e}}$ éd., Paris, M.-T.-H. Fénin, 1954, p. 76-90.

I46 P. Deyon, Le mercantilisme, Paris, Flammarion, 1969, p. 58.

I47 Une première citation offre une vision synthétique du changement de modèle qui se propage : « La nature donc, ou la Providence, peut seule faire observer cette justice pourvu encore une fois que qui que ce soit autre ne s'en mêle; et voici comme elle s'acquitte. Elle établit d’abord une égale nécessité de vendre et d’acheter dans toutes sortes de trafics, de façon que le seul désir de profit soit l'âme de tous les marchés, tant dans le vendeur que dans l'acheteur ; et c'est à l'aide de cet équilibre ou de cette balance, que l'un et l'autre sont également forcés d'entendre raison, et de s'y soumettre. La moindre dérogeance, sans qu'il importe dans lequel des deux, gâte aussitôt tout [...] », P. de Boisguilbert, Dissertation sur la nature des richesses: où l'on découvre la fausse idée qui règne dans le monde à l'égard de ces trois articles, [En ligne], Institut Coppet, 2014, p. 33.

I48 A. Béraud, G. Faccarello et al., Nouvelle histoire de la pensée économique. Des scolastiques aux classiques, vol. I, Paris, La Découverte, I992, p. 164-I74.

I49 F.-V.-D. de Forbonnais, Élemens du commerce, t. 2, $2^{\mathrm{e}}$ éd., Leyde, Briasson, David l'aîné, le Breton et Durand, I754, p. I38226.

I50 Les précurseurs comme Mun ou Child estiment que les échanges doivent être stimulées par la circulation de la monnaie qui doit être libéralisée. Law et Melon considèrent que la circulation de l'argent est l'instrument nécessaire pour que les acteurs économiques prospèrent, contrairement à la thésaurisation, G. Poulalion, Histoire de la pensée économique : des origines à la fin du XIX $X^{e}$ siècle, $\mathrm{I}^{\text {er }}$ éd., Lyon, l'Hermès, I993, p. II5.

I5I C. Gide et C. Rist, Histoire des doctrines économiques : depuis les physiocrates jusqu'à nos jours, 6e éd., Paris, Dalloz, 20oo, p. II-2I et I3I-I33; D. Villey, op. cit., p. I06-III. Cette modification de la pensée économique s'accompagne d'une nouvelle génération d'économistes qui accèdent au pouvoir, voir D. Margairaz, «La formation du réseau des foires et des marchés : stratégies, pratiques et idéologies », Annales. Économies, sociétés, civilisations, 41/6, 1986, p. 1215-1242.

I52 T.-W. Angell, The Theory of International Prices. History. Criticism and Restatement, Cambridge, Harvard University Press, 1926, p. 204. 
est le seul à accuser la limitation du circuit retour de porter atteinte aux intérêts du négoce et à l'équité ${ }^{153}$. C'est également l'unique auteur dont la préface dénonce les insuffisances de la législation, mais aussi de la doctrine ${ }^{154}$ ! Il est possible d'y voir les paroles d'un juriste du prétoire, dont la formation et la carrière sont antérieures à l'ordonnance de ${ }_{1673}$, et dont l'ouvrage jouxte les mutations juridiques qui sont liées à l'enseignement du droit français à la fin du XVIIe siècle. Ainsi, Dupuis s'inscrit entre deux époques. Il reste largement à l'écart de la génération des commentateurs, mais il bénéficie d'un regard critique sur la législation tout en étant encore très marqué par la production doctrinale des anciens spécialistes du droit des marchands.

67. À l'exception de cette discrète incursion, les juristes font preuve de discipline et s'intègrent parfaitement à la conclusion tirée par Dina Ribard à propos de la production doctrinale majoritaire de la seconde modernitérss :

On voit que si l'acte de recueillir, de rassembler et de rapprocher des lois sans y ajouter de discours extérieur au leur propre est donné pour la meilleure manière de les rendre compréhensibles, à l'usage de ceux qui les étudient comme surtout de ceux qui doivent les appliquer, c'est parce qu'ainsi mises en recueil, les lois elles-mêmes montrent les infléchissements et "tempéraments" qu'elles doivent recevoir dans l'exécution. Ce qui pourrait être une explication $[\ldots]$ n'en est donc pas une $[\ldots]$.

68. Sous cet angle, on perçoit que la transformation de la pensée juridique moderne, constatée chez la majorité de nos auteurs, éloigne les juristes de la question économique pour en faire peu à peu de purs techniciens du droit ${ }^{156}$. Dès lors, si la frontière entre l'École et le Palais est résiduelle, celle entre le droit et le commerce est plus épaisse. Les ouvrages étudiés, à l'exception de ceux de la famille Savary, sont avant tout destinés aux praticiens du droit, à la mise en ouvre des prescriptions de l'ordonnance de 1673 pour la résolution des litiges. Même lorsque le négoce est le lectorat visé, l'objectif reste circonscrit à la même logique. Chez un auteur comme Rogue, l’idée est développée dès sa préface puisque, rappelons-le, ce dernier entend faciliter la compréhension des lois du commerce pour réduire les litiges portés devant les juges-consuls ${ }^{157}$. Parmi les professeurs de droit français, comme Jousse et Pothier ${ }^{158}$, leur méthode rationnelle d'analyse et d'enseignement repose sur la concision et la clarté des discours. Cette approche est peu compatible avec l'exploration des causes et des solutions économiques aux problèmes du voyage retour de l'argent.

69. Alors que nos auteurs entretiennent un lien profond avec la pratique, seulement trois d'entre eux disposent d'une proximité avec celle du commerce : il s'agit des membres de la famille Savary 159 . Dès lors, la distance avec le monde des affaires peut constituer une piste de réflexion pour expliquer les différentes lacunes observées, malgré d'indéniables réussites ${ }^{160}$. La plupart des juristes écrivent à destination des praticiens du droit, non des négociants eux-mêmes ${ }^{161}$. Le problème est que, malgré des frontières parfois poreuses, la pratique commerciale ne renvoie pas aux mêmes réalités que la pratique juridique. Dans les deux cas il s'agit du même instrument qui est en cause, en l'occurrence la lettre de change. Mais la façon de l'appréhender diffère d'une situation à l'autre. D’une part, le négoce s'intéresse avant tout à sa mise en

I53 J. Dupuis, op. cit., p. 172-173.

154 Supra $\$ 2$ I.

I55 D. Ribard, art. cit., p. II6.

I56 J.-L. Thireau, « Jurisconsulte », Dictionnaire de la culture juridique, dir. D. Alland et S. Rials, Paris, Presses Universitaires de France, 2003, p. 879.

157 Supra $\$ 2$ I.

I5 8 L'aspect pratique est central dans ses écrits, la réforme du droit est un sujet qui l'intéresse peu, A. Monti, « Le rôle et les pouvoirs du juge dans l'œuvre de Daniel Jousse », Daniel Jousse, op.cit., p. 40-4I. Pothier ne souhaite pas mêler des arguments extra-juridiques à ses explications, notamment pour ne pas alourdir son propos, voir la préface de JeanLouis Halpérin dans R.-J. Pothier, Traité des obligations, op. cit.

I59 Boutaric mis à part, car il se détourne rapidement de la voie prise par sa famille dans le négoce au profit du droit, J. Poumarède, « Boutaric, François (de) », Dictionnaire historique, op. cit., p. 165-166.

I60 L'hypothèse a déjà été soulevée par A. Mages, art. cit., p. 238.

I6I Sur ce point, J.-P. Lévy, «Les actes de la pratique, expression du droit », Revue historique de droit français et étranger, 66/2, avril-juin 1988, p. I5I-170 ; J. Hilaire, La vie du droit, Paris, Presses Universitaires de France, 1994, p. 270 et suivantes, mais aussi F. Terré, Du juridique et du social, Paris, Mare \& Martin, 2012, p 190-230. 
œuvre dans les échanges, aux résultats économiques qu'elle peut produire. D’autre part, les professionnels $\mathrm{du}$ droit appréhendent son régime juridique et la résolution des litiges qui la concerne devant les tribunaux. Cette dichotomie est confirmée par la formation des jeunes négociants. Les ouvrages juridiques sont largement concurrencés par l'apprentissage au comptoir et dans des manuels spécialisés ${ }^{162}$ où la question des circuits du change et du rechange y est bien mieux traitée ${ }^{163}$, avec une visibilité plus large sur les débouchés étrangers et une méthode savante de calcul des bénéfices par la règle de trois ou la règle conjointe ${ }^{164}$.

70. Toutefois, les juristes ne sont pas les seuls à subir consciemment ou non cet immobilisme. En effet, la physiocratie, pour citer le courant le plus emblématique de la fin du XVIII siècle et qui s'oppose aux tendances mercantilistes, ne reçoit pas une adhésion unanime ${ }^{165}$, malgré une propagation des idées économiques de plus en plus vive dans le grand public ${ }^{166}$. Sa diffusion progresse en Europe, mais elle rencontre certains obstacles au sein des institutions françaises, notamment à la suite de l'échec du système de $\operatorname{Law}^{167}$.

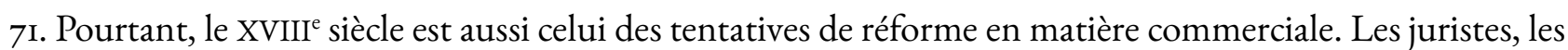
négociants, tout comme certains agents royaux, manifestent une volonté d'améliorer ou de remplacer l'ordonnance de $1673^{168}$. C'est un mouvement commun qui s'affirme dès 1700 , mais dont la mise en ouvre ne commence véritablement qu'en 1778 sous l'initiative du garde des Sceaux ${ }^{169}$. Entre temps, l'agitation se répand dans différents domaines mercantiles et s'accentue dans les pratiques du change. Par exemple, en I7II, la question d'une possible dispense de protêt est soulevée devant la chambre du commerce de Dunkerque $^{170}$. En 176 I, devant la même chambre, est débattue celle du nombre des parties au contrat de change ${ }^{171}$. La chambre de Rouen émet également un mémoire en 1716 pour la modification de l'ordonnance. Toutefois, ce projet ne vise que trois modifications du titre 6 de la législation, concernant les

I62 Par exemple, voir J. Meuvret, « Manuels et traités à l'usage des négociants aux premières époques de l'âge moderne », Études d'histoire économique : recueil d'articles, Paris, Armand Colin, 1971, p. 23I-250 ; J. Hoock et P. Jeannin, « Une enquête en cours, la bibliographie analytique des manuels et traités à l'usage des marchands, I470-1820 », Histoire EG Mesure, I/2, 1986, p. 85-97 et P. Jeannin, «La diffusion des manuels de marchands : fonctions et stratégies éditoriales », Revue d'histoire moderne et contemporaine, 45/3, juillet-septembre 1998, p. 515-557. Concernant l'apprentissage au comptoir, voir par exemple P. Butel, « Comportements familiaux dans le négoce bordelais au XVIII siècle », Annales du Midi: revue archéologique, historique et philologique de la France méridionale, 88/127, 1976, p. 139-157 et F. Cadilhon, « Les négociants aquitains et la diffusion de l'enseignement des langues étrangères aux XVIII ${ }^{\mathrm{e}}$ et XIX $\mathrm{X}^{\mathrm{e}}$ siècles $\gg$, Annales du Midi: revue archéologique, historique et philologique de la France méridionale, I0I/188, 1989, p. 449-456.

163 Nous pouvons ici prendre pour témoins T. de Bléville, Le Banquier françois ou la Pratique des lettres de change, Paris, Musier, 1724 ; C. Irson, Pratique generale et methodique des changes etrangers, Paris, Jombert, 1687 ou encore S. Ricard, Le Nouveau négociant, Bordeaux, Boé, 1686.

I64 Sans pour autant masquer les lacunes de ces manuels sur le régime juridique, apanage des juristes.

I65 La place des monopoles contrebalance l'élan de liberté en matière économique. Les grandes institutions commerciales sont animées par cette ambivalence qui entretient un attachement aux grands principes du mercantilisme. Par exemple, voir P. Butel, Histoire de la Chambre de Commerce et d'Industrie de Bordeaux des origines à nos jours (1705-1985), Bordeaux, Chambre de Commerce et d'Industrie de Bordeaux, 1988, p. 86-93.

I66 Le XVIII siècle est favorable à la diffusion des idées économiques au sein de certains cercles intellectuels, mais aussi au niveau de la société dans son ensemble. Les physiocrates s'emparent très tôt de cette brèche pour propager leurs théories, notamment à l'aide de périodiques et de dictionnaires, J. Hoock, «Information commerciale et économique. De l'encyclopédie commerciale à la presse économique, 1680-1820 », L'information économique, XVI $I^{e} X I X^{e}$ siècle: journées d'études du 21 juin 2004 et du 25 avril 2006, dir. D. Margairaz et P. Minard, Paris, Comité pour l'histoire économique et financière de la France, 2008, p. 65-66.

I67 T. Carvalho, La physiocratie dans l'Europe des Lumières. Circulation et réception d'un modèle de réforme de l'ordre juridique et social, Thèse Droit Rennes I, vol. I, 2016, p. 38-42 et P. Harsin, Les doctrines monétaires et financières en France du XVI siècle au XVIII siècle, Paris, M. Morineau, I928, p. I2.

I68 J. Hilaire, Introduction historique, op. cit., p. 87.

I69 Sur le projet Miromesnil, voir O. Descamps et R. Szramkiewicz, op.cit., p. I86; P. Reulos, « Une source du code de commerce : le projet Miromesnil », Revue trimestrielle de droit commercial, I, 1948, p. 224-229 et H. Lévy-Bruhl, Un projet de Code de commerce à la veille de la Révolution, le projet Miromesnil (1778-1789), Paris, 1932.

I70 A. Vandenbossche, « Note sur la dispense du protêt faute de paiement de la lettre de change aux XVII et XVIII siècles », Mélanges offerts à Jean Brèthe de La Gressaye, Bordeaux, Éditions Bière, 1967, p. 791-796.

I7I Id., «Un Mémoire de la chambre de commerce de Dunkerque sur la lettre de change (176I) 》, Mélanges de droit, d'bistoire et d'économie offerts à Marcel Laborde-Lacoste, Bordeaux, Éditions Bière, 1963, p. 435-459. 
articles i et 7 , le rechange n'est donc pas concerné ${ }^{172}$. Malgré l'intérêt porté à la question cambiaire, ces diverses manifestations intéressent avant tout le régime juridique de la lettre de change et laissent la question économique du rechange et de ses circuits à l'écart.

Il faut attendre le projet Miromesnil pour que le voyage retour de l'argent soit concerné. Il n'est toutefois abordé qu'indirectement, au titre de la suppression de la remise de place à place parmi les mentions obligatoires devant figurer sur la lettre ${ }^{173}$. Cette prévision rend alors caduque toute justification liée au circuit du change et du rechange, modifiant considérablement les prescriptions de l'ordonnance. Toutefois, l'épisode révolutionnaire vient suspendre cette question et laisse inachevée toute tentative de réforme du droit cambiaire.

\section{Victor LE BRETON-BLON}

Doctorant en Histoire du Droit

Université de Bordeaux, Institut de Recherche Montesquieu - EA 7434

v.lebretonblon@gmail.com

I72 Id., Un projet de Code de commerce sous la Régence, Paris, Économica, 1980.

I73 M.-A.-M., Darwish, op. cit., p. 42-43. 